\title{
Transition to invasive breast cancer is associated with progressive changes in the structure and composition of tumor stroma
}

Tyler Risom ${ }^{1}$, David R Glass ${ }^{1}$, Candace C Liu ${ }^{1}$, Belén Rivero-Gutiérrez ${ }^{1}$, Alex Baranski ${ }^{1}$, Erin F McCaffrey $^{1}$, Noah F Greenwald ${ }^{1}$, Adam Kagel ${ }^{1}$, Siri H Strand ${ }^{1}$, Sushama Varma ${ }^{1}$, Alex Kong ${ }^{1}$, Leeat Keren $^{1}$, Sucheta Srivastava ${ }^{1}$, Chunfang Zhu ${ }^{1}$, Zumana Khair ${ }^{1}$, Deborah J Veis ${ }^{5}$, Katherine Deschryver ${ }^{2}$, Sujay Vennam ${ }^{1}$, Carlo Maley ${ }^{4}$, E Shelley Hwang ${ }^{3}$, Jefferey R Marks ${ }^{3}$, Sean C Bendall ${ }^{1}$, Graham A Colditz ${ }^{2}$, Robert B West ${ }^{1 *}$, Michael Angelo ${ }^{1 *}$

${ }^{1}$ Stanford University School of Medicine, Department of Pathology; ${ }^{2}$ Washington University School of Medicine, Department of Surgery; ${ }^{3}$ Duke University, Department of Surgery; ${ }^{4}$ Arizona State University, Biodesign institute; ${ }^{5}$ Washington University School of Medicine, Departments of Pathology \& Immunology

${ }^{*}$ co-corresponding authors

\section{Abstract}

Ductal carcinoma in situ (DCIS) is a pre-invasive lesion that is thought to be a precursor to invasive breast cancer (IBC). To understand how the tumor microenvironment (TME) changes with transition to IBC, we used Multiplexed lon Beam Imaging by time of flight (MIBI-TOF) and a 37-plex antibody staining panel to analyze 140 clinically annotated surgical resections covering the full spectrum of breast cancer progression. We compared normal, DCIS, and IBC tissues using machine learning tools for multiplexed cell segmentation, pixel-based clustering, and object morphometrics. Transition from DCIS to IBC was found to occur along a trajectory marked by coordinated shifts in location and function of myoepithelium, fibroblasts, and infiltrating immune cells in the surrounding stroma. Taken together, this comprehensive study within the HTAN Breast PreCancer Atlas offers insight into the etiologies of DCIS, its transition to IBC, and emphasizes the importance of the TME stroma in promoting these processes. 


\section{Introduction}

39 Ductal Carcinoma in situ (DCIS) is a preinvasive lesion where tumor cells within the breast duct are isolated from the surrounding stroma by a near-continuous layer of myoepithelium and basement membrane proteins. This histologic feature is the central

42 property that distinguishes it from invasive breast cancer (IBC), where this barrier has broken down and tumor cells have invaded the stroma (Figure 1A).

DCIS comprises $20 \%$ of new breast cancer diagnoses, but unlike IBC, in itself is not a life-threatening disease. However, if left untreated, up to half of these patients will develop IBC within 10 years (Betsill et al., 1978; Erbas et al., 2006; Eusebi et al., 1994; Page et al., 1982; Ryser et al., 2019).

Sequencing-based approaches have been used extensively over the last decade to identify molecular features that could elucidate the connection between DCIS and IBC. Genomic profiling has identified recurrent copy number variants (CNV) that are more prevalent in high grade DCIS lesions (Afghahi et al., 2015; Buerger et al., 1999; Fujii et al., 1996). Meanwhile, comparison of paired DCIS and IBC lesions from the same patient has provided clues into the clonal evolution from in situ to invasive disease by revealing genomic alterations that are acquired during this transition (Ak et al., 2018; Kim et al., 2015; Newburger et al., 2013). To date, however, these findings have not been found to consistently explain this transition. Similarly, the utility of tumor phenotyping by single-plex immunohistochemical tissue staining has been limited as well.

In light of this uncertainty, clinical management has trended towards treating all patients presumptively as progressors with surgery, radiation therapy, and pharmacological interventions that carry risks for therapy-related adverse events.

61 Consequently, this approach is likely to be overly aggressive for non-progressors. Thus,

62 understanding the central biological features in DCIS that drive the transition to IBC is a 63 critical unmet need.

64 Surprisingly, despite all the information now known about the genetic and functional state of tumor cells in DCIS, histopathology remains the only reliable way to 66 diagnose it. Thus, DCIS is an intrinsically structured entity where the spatial orientation 67 of tumor, myoepithelial, and stromal cells is the primary defining feature that distinguishes it from other forms of breast cancer. 
To understand how DCIS structure and single cell function are interrelated, we use new tools previously developed by our lab for highly multiplexed subcellular imaging to analyze a large cohort of human archival tissue samples covering the spectrum of breast

72 cancer progression from in situ to invasive disease. In previous work, we used

73 Multiplexed Ion Beam Imaging by Time of Flight (MIBI-TOF) and a 36-plex antibody

74 staining panel to identify rule sets governing tumor microenvironment (TME) structure in

75 triple negative breast cancer that were highly predictive of the composition of immune 76 infiltrates, the expression of immune checkpoint drug targets, and 10-year overall survival 77 (Keren et al., 2018).

This effort provided a framework for how TME structure and composition could be used more generally as a surrogate readout to understand the functional response to neoplasia. With this in mind, we sought to determine to what extent similar features involving myoepithelial, stromal, and immune cells in the DCIS TME might play a pivotal role in breast cancer progression. Each of these have been implicated previously to promote local invasion (Barsky and Karlin, 2005; Ibrahim et al., 2020), metastasis (Pelon et al., 2020; Shani et al., 2020), and to correlate with clinical progression (Yang et al., 2018; Zhou et al., 2018).

Here, we report the first systematic, high dimensional analysis of breast cancer progression using the Washington University Resource Archival Human Breast Tissue (RAHBT) cohort: a clinically annotated set of archival tissue from patients diagnosed with DCIS and IBC. Because the DCIS patient population is complicated by differences in age, parity status, tumor subtype, and treatment course, a well-conceived cohort design is crucial for identifying meaningful features amidst these confounding variables. In light

92 of this, the RAHBT cohort was composed of primary DCIS tumors from women who later

93 progressed to invasive disease that were age and year-of-diagnosis matched with control 94 tissue from women with DCIS that did not recur.

We used MIBI-TOF and a 37-plex antibody staining panel to comprehensively define the cellular composition and structural characteristics in 122 of these samples,

97 which included normal breast, DCIS, and recurrent IBC samples. We applied machine 98 learning tools for multiplexed cell segmentation and spatial analytics to enumerate 16 cell populations and to quantify how these populations are spatially distributed relative to one 
100

101

102

103

104

105

106

107

108

109

110

111

112

113

114

115

116

117

118

119

120

121

122

123

124

125

126

127

128

another. Object morphometrics and high dimensional pixel clustering were used to annotate the structure of stromal collagen and to discover new myoepithelial phenotypes that track with disease progression. These findings were corroborated by transcriptomic data acquired on coregistered tissue regions isolated by laser capture microdissection.

We systematically compared these features to understand how different phenotypic and structural properties of the DCIS TME change with progression to IBC. $\mathrm{BC}$ progression was typified by a reduction in myoepithelial integrity, a shift in fibroblast function towards proliferative cancer-associated states (CAFs), remodeling of collagen in the extracellular matrix (ECM), and a compositional and spatial reorganization of the immune microenvironment. We used the 1,093 features quantified in these analyses to build a random forest classifier for predicting which patients would later progress to invasive disease based exclusively on the original diagnostic biopsy. This classifier demonstrated an AUC of 0.83 and was heavily weighted for stromal features that were reliant on spatial information. Taken together, this work provides new insight into potential etiologies of DCIS progression that will guide development of future diagnostics and serve as a template for how to carry out similar analyses of preinvasive cancers.

\section{Results}

\section{A multiplexed imaging interrogation of DCIS progression to invasive disease}

We examined the transition from DCIS to IBC by profiling accumulative changes in the phenotype, structure, and spatial distribution of myoepithelium, tissue stroma, and immune cells in archival formalin-fixed paraffin-embedded (FFPE) patient tissue of three distinct progression groups: normal breast $(n=9)$, IBC $(n=16)$, and DCIS $(n=$ 115). These IBC samples were disease recurrences from women with a prior diagnosis of DCIS. Of the $115 \mathrm{DCIS}$ samples, 78 were RAHBT patients with a new diagnosis and no signs of IBC (pure, primary), while 14 were pure DCIS recurrences (pure, recur)(Figure $1 \mathrm{~A}$, Table S1). The remaining 23 patients comprised a third group of synchronous lesions 

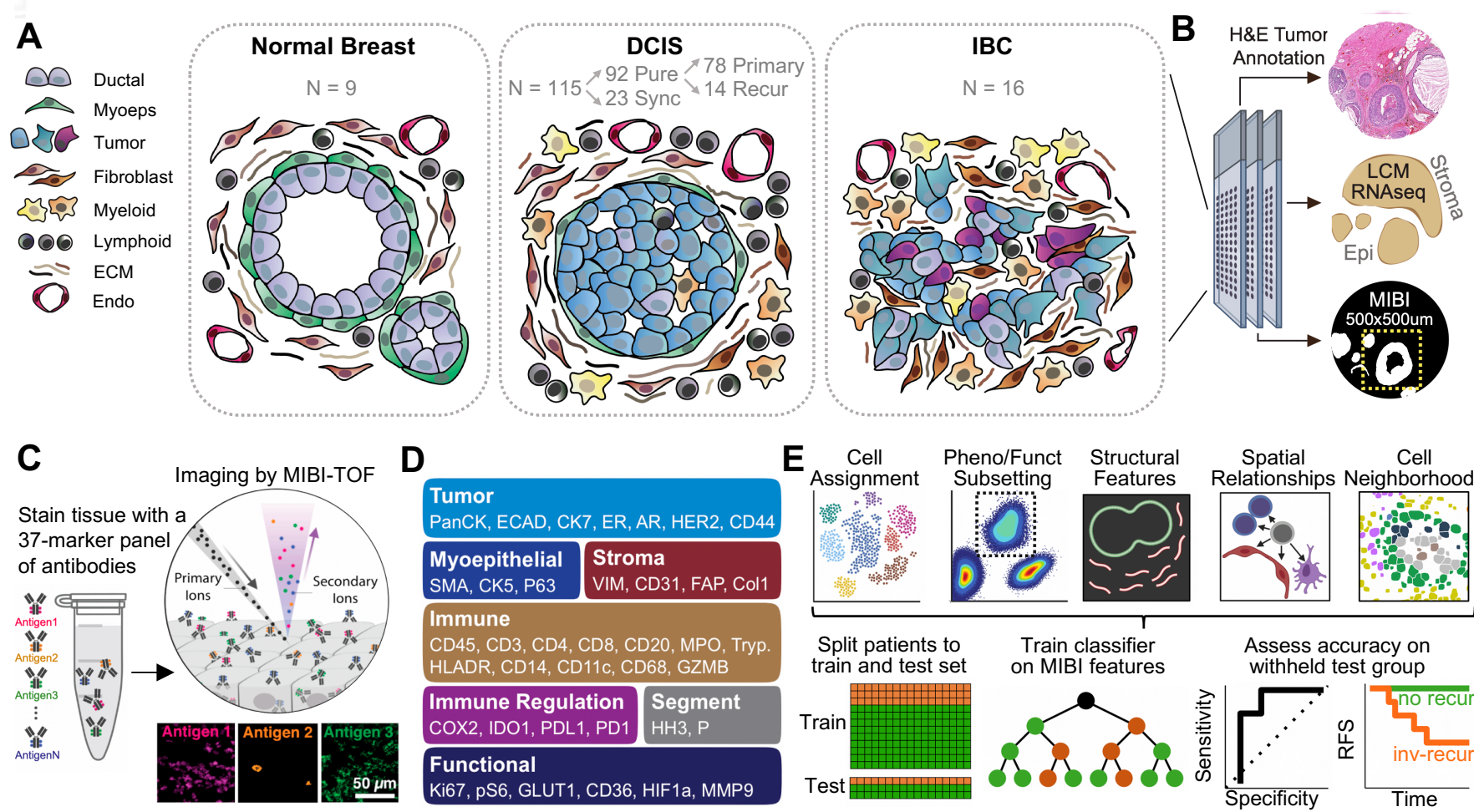

Spatial Cell
Relationships Neighborhoods

Figure 1. A multiplexed Imaging Interrogation of DCIS Progression to Invasive Disease

A. Schematic depicting the tumor stages and patient sample numbers profiled in this study, including normal breast, pure DCIS (primary or recurrent), synchronous DCIS (Sync), and invasive breast carcinoma (IBC). B. Depiction of the parallel tissue analysis methods used in this study including H\&E, laser capture microdissection (LCM) RNAseq, and MIBI-TOF. C. Overview of the MIBI-TOF workflow. D. Markers used in the MIBI-TOF panel are displayed, grouped by target cell type or protein class. E. Workflow showing feature types extracted from the MIBI-TOF analysis that were used to train a random forest classifier to differentiate DCIS samples with or without risk of recurrence.

129 procured at Stanford Hospital where both DCIS and IBC were identified in different parts 130 of the tissue at the time of diagnosis (Sync). For this set of patients, only the in situ 131 component was analyzed.

$1321.5 \mathrm{~mm}$ cores of each tumor were arranged in tissue microarrays (TMAs). Three adjacent sections were then used for 1) H\&E staining and annotation by a pathologist, 2)

134 RNA transcriptome analysis of ductal and stromal regions isolated using laser-capture 135 microdissection (LCM-Smart-3SEQ)(Foley et al., 2019), and 3) highly multiplexed 136 imaging by MIBI-TOF of a $500 \times 500 \mu m$ field-of-view (FOV)(Figure 1B). By ensuring that 137 each of these analyses were spatially coregistered with one another, the proteomic and 138 transcriptomic features revealed by MIBI-TOF and LCM-RNAseq could be directly 139 correlated to understand the interplay between single cell composition and global 140 transcriptional programs. 
For MIBI-TOF, we constructed a 37-plex staining panel of metal-conjugated antibodies that would permit us to: 1) map the lineage and spatial location of every cell, 2) identify lineage subsets of tumor, fibroblasts, and immune cells previously implicated in BC progression, and 3) characterize the composition, integrity, and morphology of myoepithelium and collagen (Figure 1D, Table S2). The panel also included 11 functional markers for annotating proliferation, activation, hypoxic signaling, as well as markers

147 implicated in cancer immunoregulation, including PD-L1, IDO1, COX2 and PD1 (Figure S1). The features extracted in this analysis were then used to train a random forest classifier for predicting long term outcome (Figure 1E).

\section{A single cell phenotypic and spatial atlas of DCIS}

152 The workflow outlined in Figure 1 enabled high-dimensional, subcellular imaging of 153 dozens of proteins that recapitulated the tissue architecture observed in H\&E (Figure 154 2A). Multiplexed imaging data were processed with a low-level pipeline prior to single155 cell segmentation (Figure 2B, Figure S2B)(Keren et al., 2018; McCaffrey et al., 2020;

156 Moen et al., 2019; Valen et al., 2016), which identified on average $~ 924$ cells in each 157 FOV $(s d=317)$. To determine cell location with respect to canonical histological features, 158 we demarcated duct, stroma, and myoepithelial regions of each image based on combinatorial marker expression (Figure 2B bottom-right). Importantly, throughout this work we will be presenting cellular data either as the frequency of a parental lineage across the entire image (e.g., macrophages as \% of total immune cells) or as a cell density within a particular compartment of the image (e.g., 50 fibroblasts $/ \mathrm{mm}^{2}$ of stroma).

Hierarchical application of the FlowSOM algorithm (Van Gassen et al., 2015) was employed to identify 16 unique cell subsets in the dataset amongst the epithelial, stromal, and immune lineages (Figure 2B, S2B). Altogether, we assigned 95\% ( $n=127,451$ single cells) of cells to one of these subsets that in aggregate ranged in frequency from 0.7$56 \%$. These data were used to generate cell phenotype maps (CPM) where each cell is colored according to its subset assignment. CPM images illustrated focal enrichment of lymphocytes (Figure 2C “1"), endothelial-associated immune phenotypes (Figure 2C, "2") 
bioRxiv preprint doi: https://doi.org/10.1101/2021.01.05.425362; this version posted January 6, 2021. The copyright holder for this preprint (which was not certified by peer review) is the author/funder, who has granted bioRxiv a license to display the preprint in perpetuity. It is made available under aCC-BY-NC-ND 4.0 International license.

A
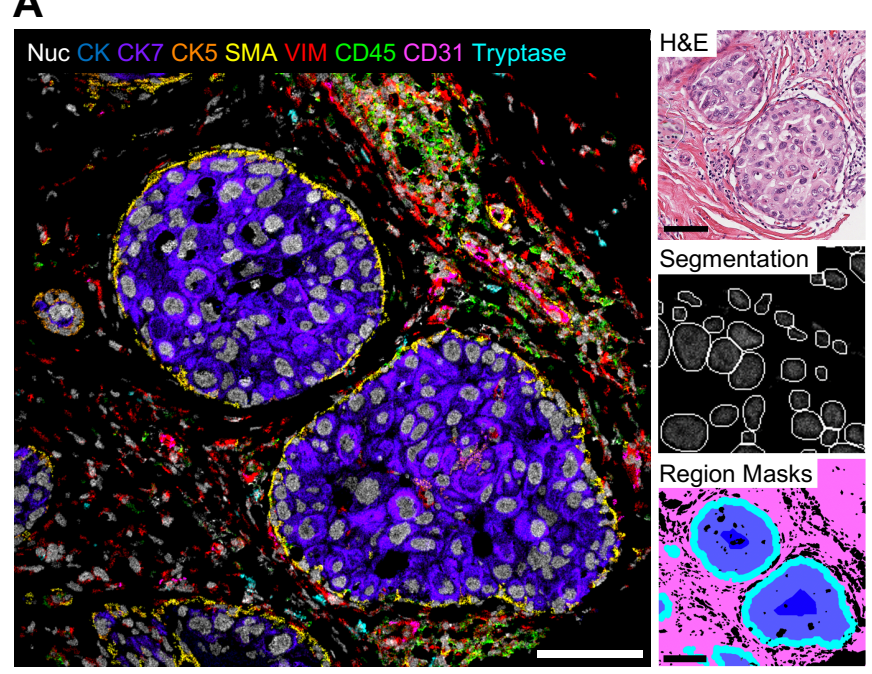

B

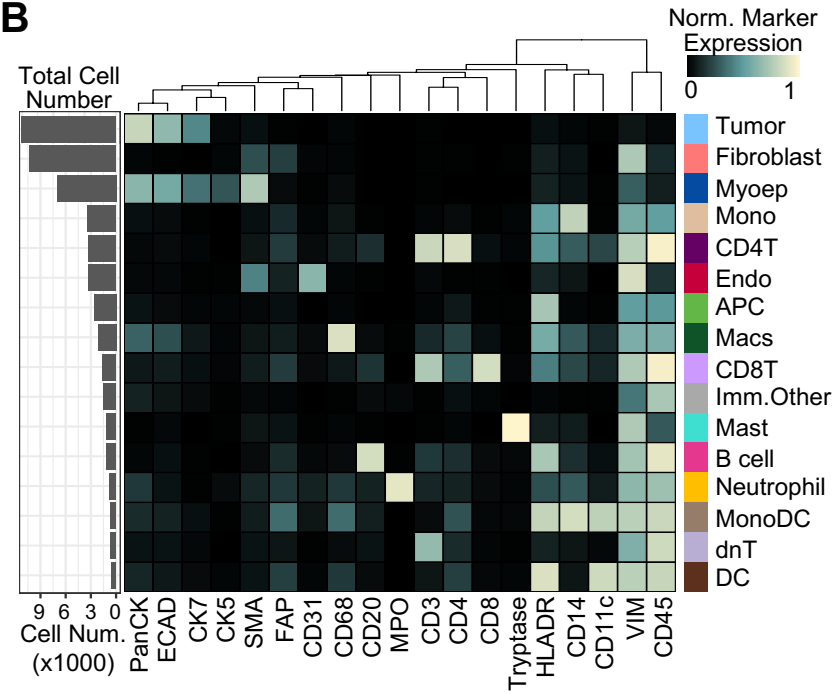

C

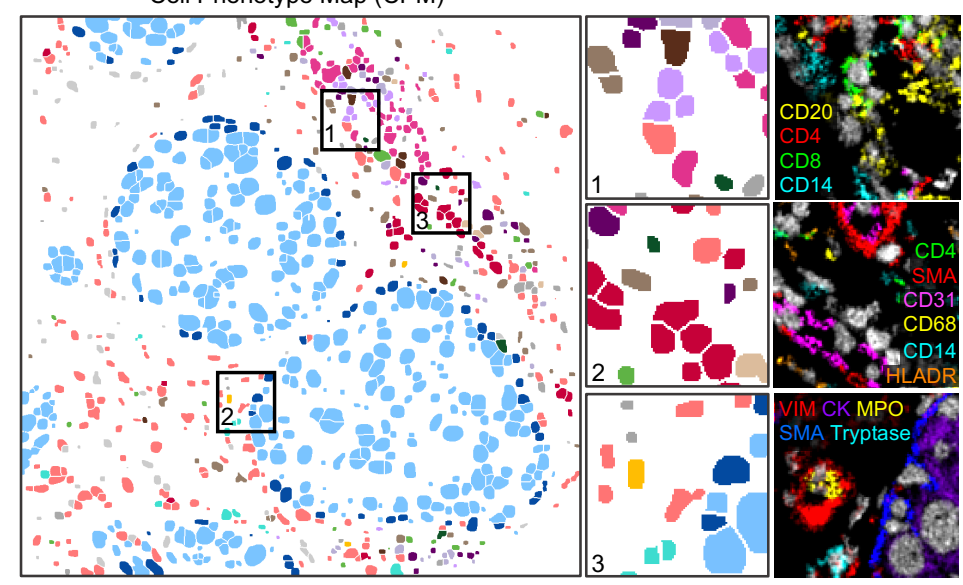

E

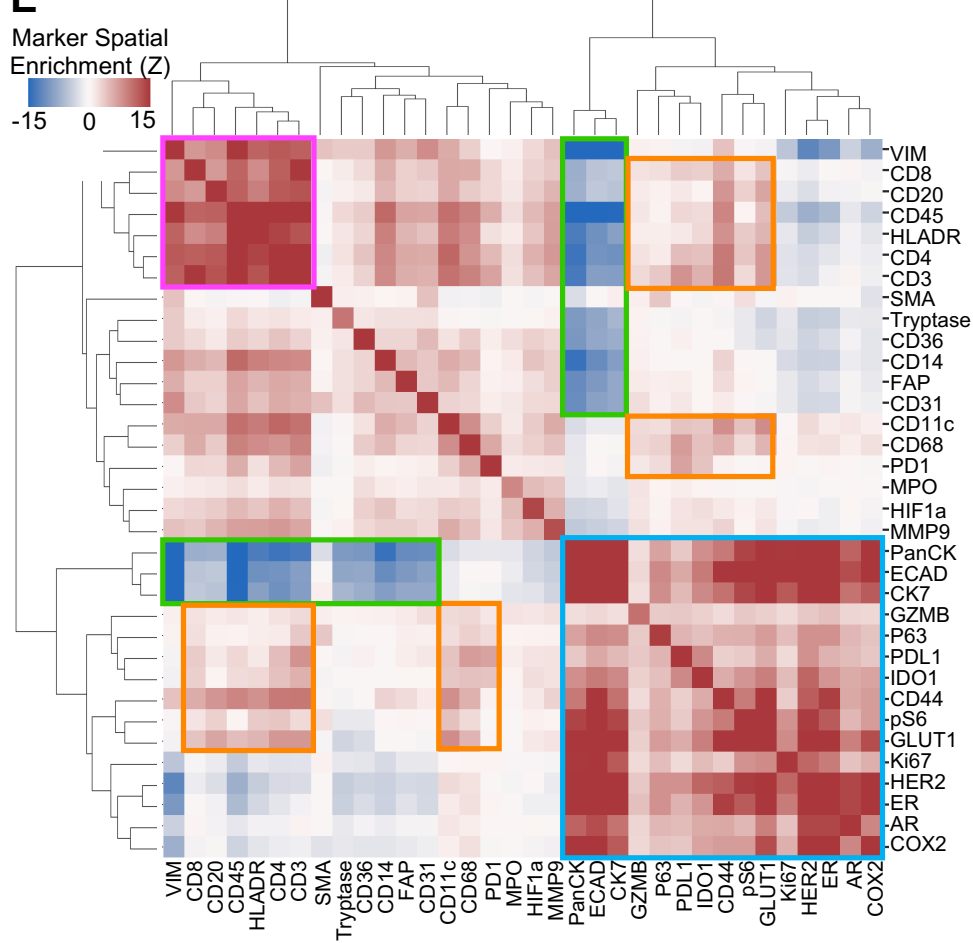

D

Tumor Myoep Fibroblast Endo Macs Mono DC MonoDC CD8-T CD4-T B cell Mast APC Neut DN-T ImmuneOther

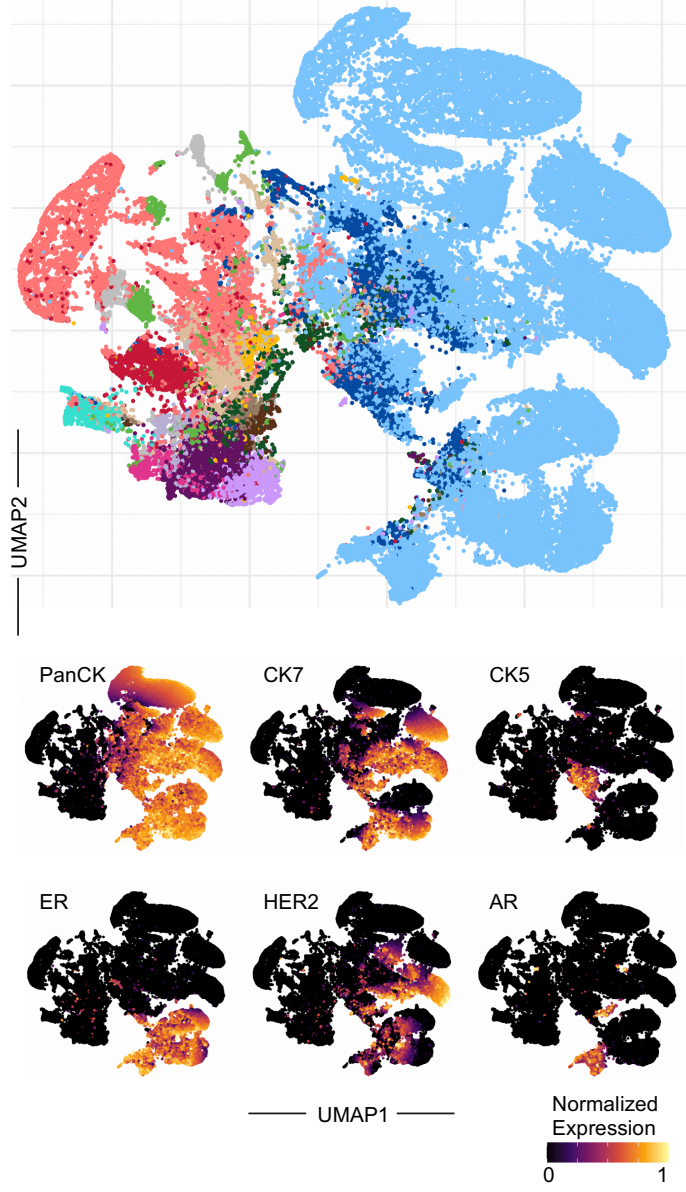




\section{Figure 2. A single cell phenotypic and spatial atlas of DCIS}

A. Representative MIBI image overlay of a DCIS tumor with a 9-marker overlay of major cell lineage markers (left) and the corresponding H\&E image (top right), example of cell segmentation (middle right), and example of region masks marking stroma (pink), myoepithelial (cyan) and ductal (blue) area, scale bars $=100 \mu \mathrm{m}$. B. Cell lineage assignments based on normalized expression of lineage markers (heatmap columns), rows are ordered by absolute abundance shown in the bar plot (left), while columns are hierarchically clustered (euclidean distance, average linkage). C. A cell phenotype map (CPM) showing cell identity by color, as defined in $F$, overlaid onto the segmentation mask. Zoomed insets with adjacent MIBI overlays show diverse lymphoid rich regions (1), endothelial-associated immune cells (2) and rare subsets like neutrophils and mast cells near ducts (3). D. UMAP visualization of all cell type populations in DCIS tumors (top), colored by cell type as in $F$, with additional plots overlaid with the normalized expression of tumor lineage and functional markers used to delineate tumor subsets (bottom).

Tumor cells were the most abundant cell type in DCIS samples $(60 \% \pm 20$ of all cells) and were comprised of multiple subsets that were defined by variable expression

174 of the luminal and basal lineage markers (CK7 and CK5, respectively), as well as ER, 175 AR, and HER2 (Figure 2D). Since these cells are isolated by a layer of myoepithelium, 176 by definition the tissue structure of DCIS is highly compartmentalized. In order to 177 determine if our analyses were capturing this fundamental facet, we used an unbiased 178 computational approach to identify sets of proteins that colocalize or avoid one another 179 more frequently than would be expected by chance. Consistent with the 180 compartmentalized nature of DCIS, tumor cell markers were spatially enriched (PanCK, 181 ECAD, CK7, HER2, ER, AR, Figure 2E, blue box) and segregated from vascular, 182 fibroblast, and immune markers (Figure 2E, green box). With respect to the latter, 183 lymphoid markers demonstrated the most prominent spatial enrichment (Figure 2E, 184 magenta box). These analyses also revealed moderate preferential enrichment in tumor 185 positive regions for pS6, COX2, and Ki67, while immunoregulatory markers were more evenly dispersed between tumor and immune-enriched regions (Figure 2E, orange box).

\section{A tumor cell phenotypic switch marks invasive transition}

189 Tumor heterogeneity in breast cancer can manifest as variations in the level of hormone receptor expression and the degree of luminal, basal, and mesenchymal differentiation.

191 DCIS has been shown to vary across the full spectrum of both of these axes, which can 192 confound identification of conserved features correlating with clinical outcome. In order 193 to understand how this heterogeneity manifests in pure DCIS and throughout the 194 transition to invasive disease, we first examined the distribution of DCIS subtypes with 195 respect to hormone receptor status (ER, AR), HER2, and Ki67 proliferation index. These markers were robustly expressed in DCIS tumors (Figure 3A) and showed expected inter- 
patient variability. Using clinical cutoffs as a guide (Figure S3A), we subtyped tumors as Luminal A (ER ${ }^{+}$, HER2$\left.^{-}, \mathrm{Ki}^{-}\right)$, Luminal B (ER ${ }^{+}, \mathrm{HER} 2^{-}, \mathrm{Ki}^{+}$), HER2E (ER ${ }^{-}, \mathrm{HER}^{+}$), ERHER2 (ER $\left.{ }^{+}, \mathrm{HER}^{+}\right)$, and TNBC (ER-, HER2-) based on the frequency of positive cells for each marker. All subtypes were present in both DCIS and IBC, with similar numbers of luminal samples in each progression group (Figure 3B). HER2 ${ }^{+}$tumors were more predominant in DCIS, while TNBC was more prevalent in IBC (Figure S3B-C).

On comparing epithelial differentiation states in each progression group, we identified a consistent trend towards reduced luminal cell identity throughout tumor progression. Distinct phenotypic subsets of luminal $\left(\mathrm{CK} 7^{+}\right)$, basal $\left(\mathrm{CK} 5^{+}\right)$, EMT-like $\left(\mathrm{VIM}^{+}\right)$, and CK5/7-low cells were observed in the epithelial lineage (Figure 3C). While the majority of ductal cells in normal breast were consistently luminal $(84 \% \pm 11)$ (Figure 3D), the composition in DCIS varied widely between being predominantly luminal or CK5/7-low (57\% $\pm 33,36 \% \pm 33$ respectively). In comparison to normal tissue and IBC, these lesions were also enriched with a minority fraction of basal cells $(6.1 \% \pm 11.9)$. With progression to IBC, CK5/7-low cells predominate more frequently and were accompanied 212 by a relative increase in EMT-like cells that express vimentin (Figure 3E). We further 213 examined a subset of patients with high frequencies of vimentin-positive tumor cells by 214 LCM-RNAseq. Consistent with the shift to a mesenchymal phenotype captured by MIBI215 TOF, geneset enrichment analysis (GSEA) revealed upregulation of signaling pathways 216 relating to mesenchymal breast tumor histology and tumor invasion in patients with high 217 vs low frequencies of $\mathrm{VIM}^{+}$tumor cells (Hollern et al., 2018; Lien et al., 2007; Poola et al., 2005)(Figure 3F, Figure S3D).

The coordinated changes in tumor phenotype illustrate how cell differentiation 220 during BC progression may follow an orderly trajectory. To further explore this possibility, 221 we compared tumor cell functional states in pure, DCIS synchronous DCIS, and 222 IBC. Synchronous DCIS describes lesions where distinct areas of tissue contained either 223 fully encapsulated tumor cells (i.e., DCIS) or areas of local invasion (i.e., IBC) were both 224 present at the time of diagnosis, but in different areas of tissue (Figure 3G). Consistent 225 with their more aggressive behavior, DCIS tumor cells from synchronous lesions 

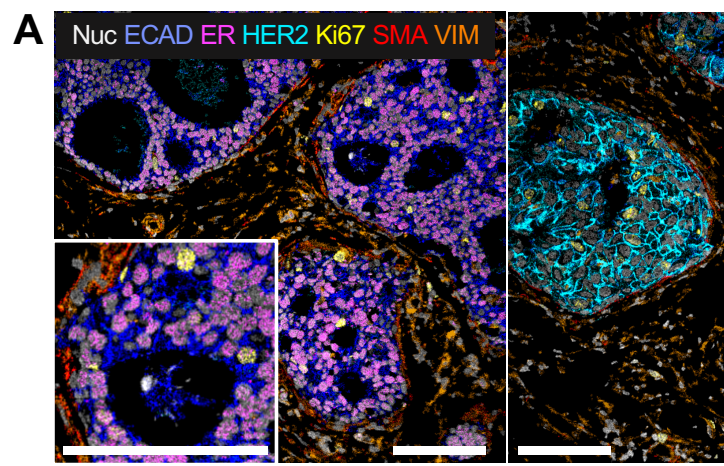

D

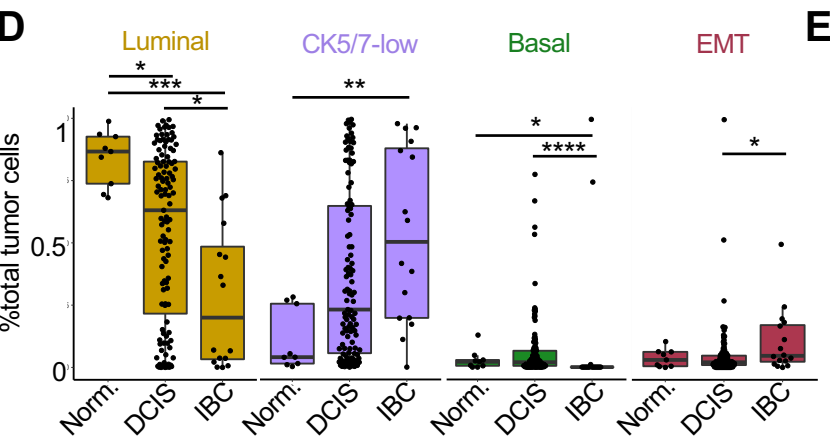

F Hollern EMT Breast Omarmor UP

NES $=-2.1$

-0.5 FDRq $=0.0014$

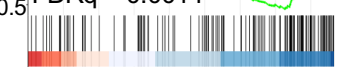

ш Lien Breast Carcinoma o- Metaplastic vs Ductal UP

NES $=-2.5$

$-0.6 \mathrm{FDRq}=0.007$

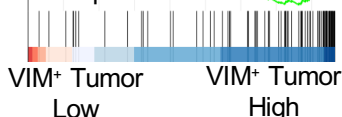

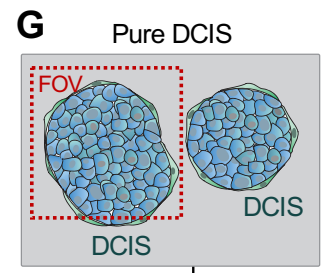

DCIS with synchronous IBC ("Sync")

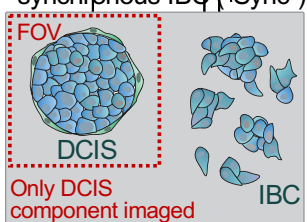

B

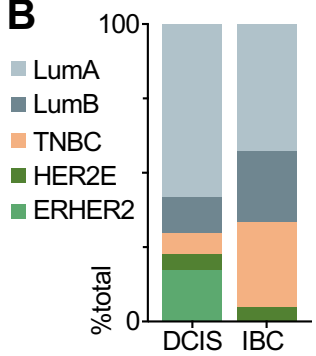

C

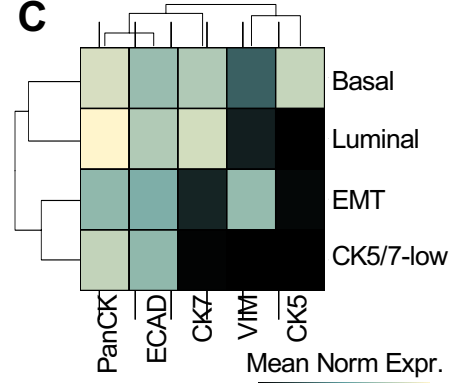

0

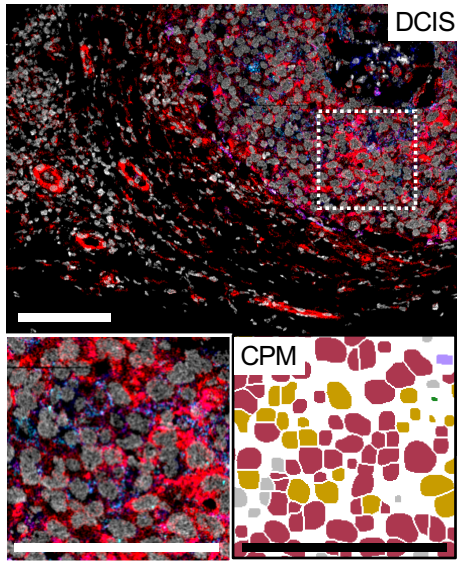

$\mathbf{H}$
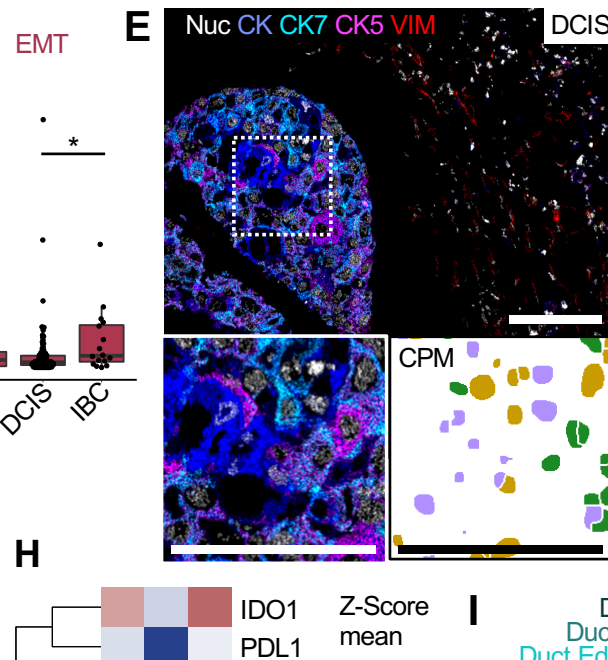

expr.

HIF1a $\quad 1.5$

pS6

Ki67

MMP9

GLUT1

$\operatorname{COX} 2$

CD36

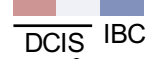

Qu" 5$)^{\circ 0}$
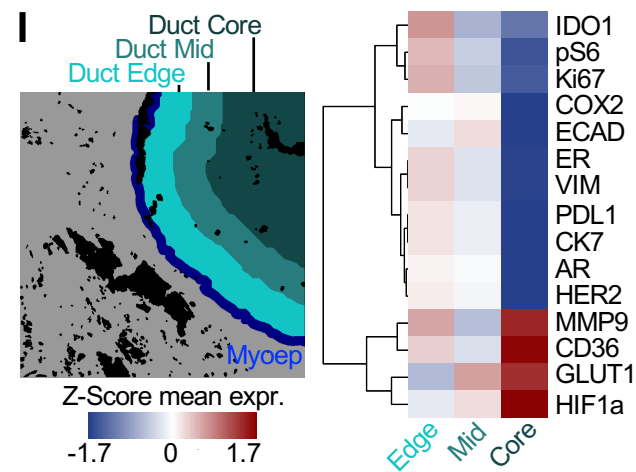

\section{Figure 3. A tumor cell phenotypic switch marks invasive transition}

A. Representative MIBI image overlays showing an ER+HER2- tumor (left) and ER-HER2+ (right), scale bars $=100 \mu \mathrm{m}$. B. Stacked barplot showing the distribution of intrinsic breast cancer subtypes in DCIS and IBC tumors, as defined by receptor expression. C. Tumor phenotype assignments based on normalized expression of markers related to markers of tumor differentiation (heatmap columns). D. Frequency of tumor differentiation states across normal breast, DCIS, and IBC. E. Representative MIBI image overlays of DCIS tumors with basal and mesenchymal features, respectively. Zoomed insets (left) with paired cell phenotype maps (right) colored by tumor phenotype identity as in $D$, scale bars $=100 \mu m$. F. Geneset enrichment analysis comparing VIM-high and low tumors with genesets related to mesenchymal tumor differentiation. G. Schematic showing the imaging FOV location in pure and synchronous DCIS tumors, which only included the DCIS component. H. Heatmap of z-score normalized functional marker expression between tumor progression groups. I. Heatmap of z-score normalized functional marker expression in DCIS tumors comparing tumor cells on the outer duct edge, tumor cells in the duct middle (duct mid), and tumor cells in the duct core.

226 demonstrated an intermediate functional profile, with features overlapping between pure

227 DCIS (GLUT1, CD36, COX2) and IBC (Ki67, pS6, HIF1 $\alpha$, MMP9) (Figure 3H). 
It is not well understood how these functional states are affected by the location of tumor cells within the duct of carcinoma in situ, where interior tumor cells far from the duct edge may have limited access to nutrients and oxygen. Interestingly, we found almost all proliferative and cell signaling molecules to be enriched in tumor cells on the duct edge, whereas HIF $1 \alpha$ and metabolite import receptors GLUT1 and CD36 were enriched in cells in the duct core, consistent with an adaptation to a low nutrient, hypoxic environment

234 (Figure 3I).

\section{Myoepithelial breakdown and phenotypic change during DCIS progression}

To understand how the structure and function of this key cellular barrier changes with progression to IBC, we next performed a targeted analysis characterizing myoepithelial cells which circumscribe both normal breast ducts and tumor cells in DCIS. Breast myoepithelium in normal tissue is a thick, highly cellular layer between the stroma and

242 ductal cells (Figure 4A). In DCIS, the myoepithelium is notably thinned out and reduced

243 in cellular density (Figure 4A-B). The remaining myoepithelial cells in DCIS tumors were 244 found to have higher proliferation relative to normal tissue, with synchronous tumors having the highest levels of the Ki67 positivity of these three groups (Figure 4C).

Given these findings, we hypothesized that loss of myoepithelial integrity (thickness $x$ percentage of duct-perimeter covered) in synchronous DCIS lesions would also be greater than in pure DCIS. To explore this question, we developed a new image analysis tool to quantify myoepithelial thickness and percent coverage of the duct edge (Figure 4D, see Myoepithelial Coverage and Thickness Analysis in Methods). This analysis revealed significant loss in myoepithelial integrity in DCIS tumors relative to normal tissue. To our surprise, however, no significant difference was observed between

254 reduction of myoepithelial cell density and myoepithelial integrity independent of the 255 presence of a neighboring invasive component.

256 After quantifying these changes in myoepithelial structure, we next sought to 257 determine how the function of this regulatory barrier is altered with disease progression. 
bioRxiv preprint doi: https://doi.org/10.1101/2021.01.05.425362; this version posted January 6, 2021. The copyright holder for this preprint (which was not certified by peer review) is the author/funder, who has granted bioRxiv a license to display the preprint in perpetuity. It is made available under aCC-BY-NC-ND 4.0 International license.
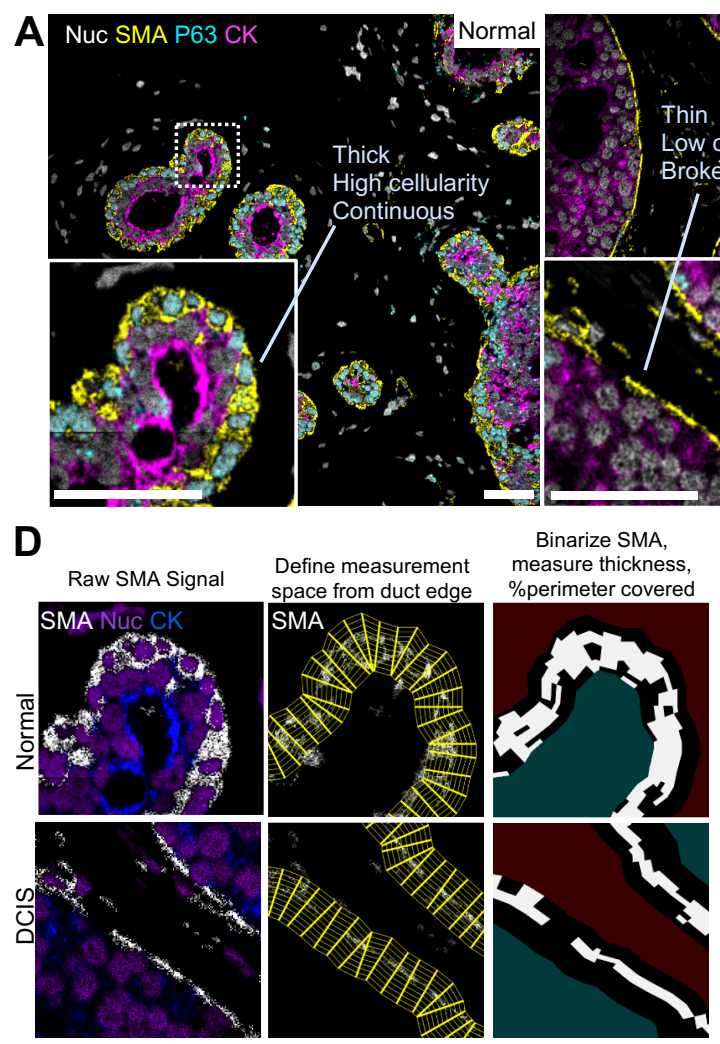

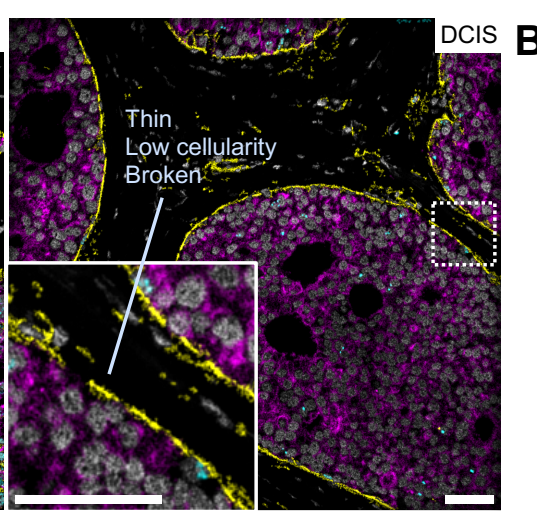

E

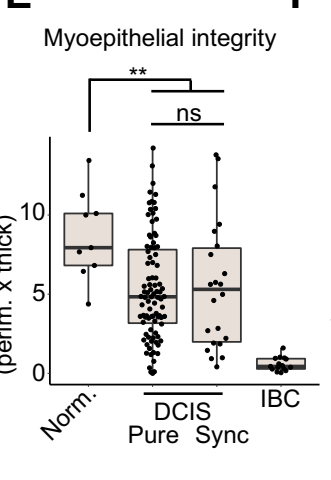

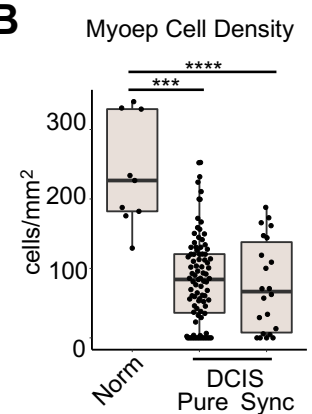

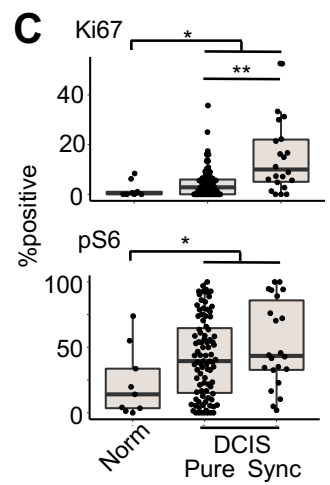

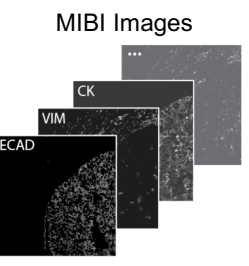

Cluster SMA+ pixels by coexpression

Apply myoepithelium mask
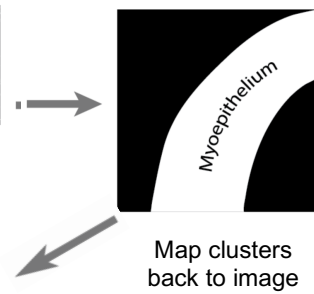

Map clusters

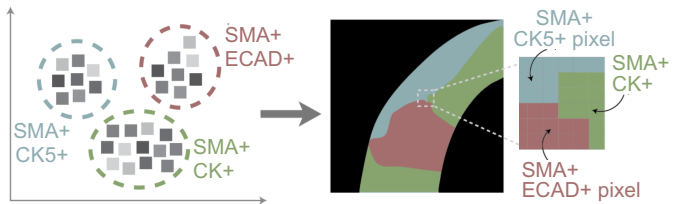

G
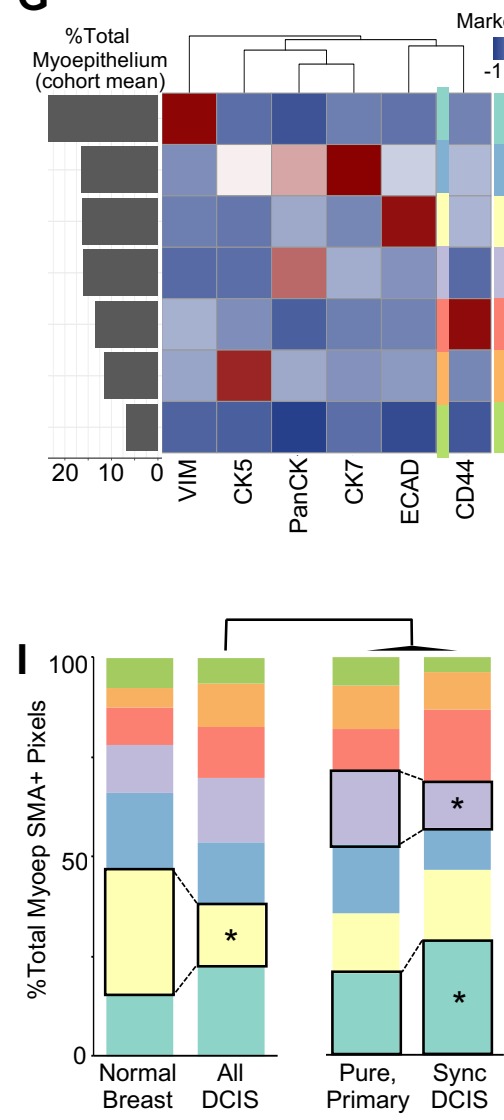

Marker Expression (Z)

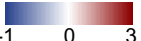

mcVIM

mcCK7

mcECAD

mcCK

mcCD44

mcCK5

mcSMAonly

.

H
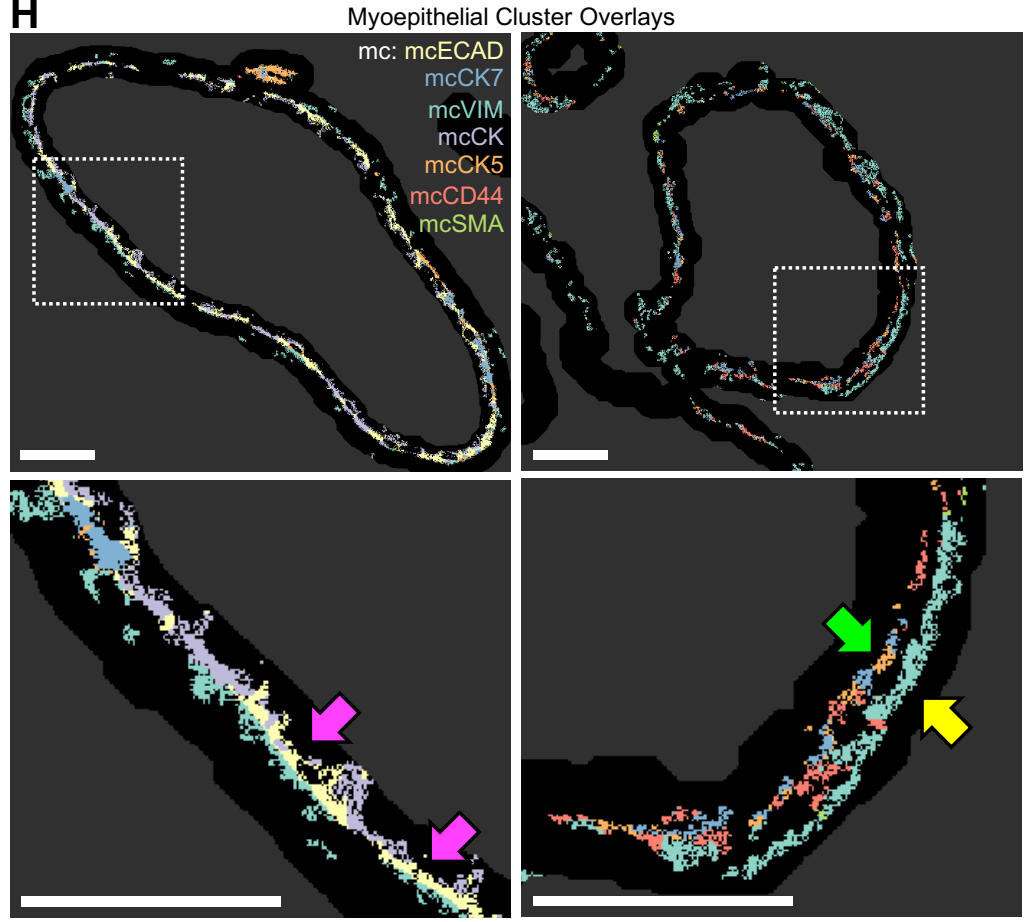

MIBI Marker Overlay
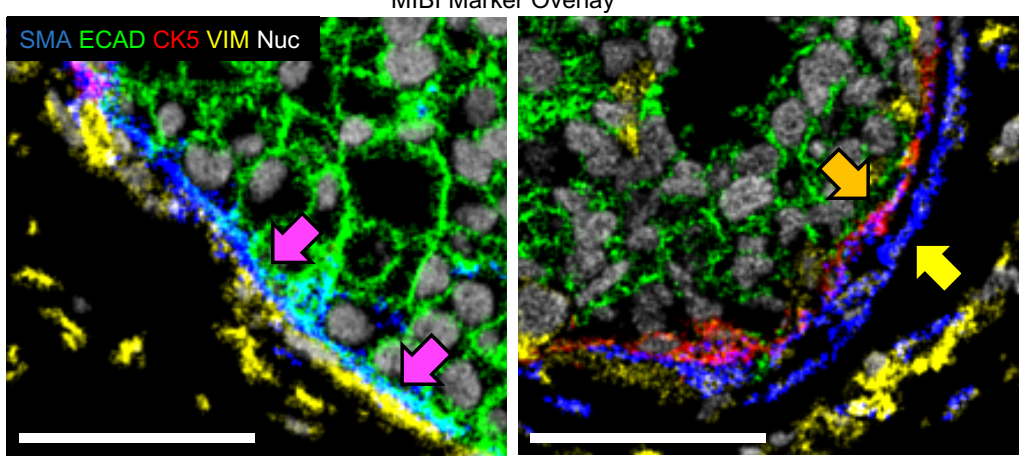
Figure 4. Myoepithelial breakdown and phenotypic change during DCIS progression

A. Representative MIBI image overlays showing SMA (yellow), p63 (cyan), and PanCK (magenta) expression in myoepithelium in normal breast (left) and DCIS (right), scale bars $=50 \mu \mathrm{m}$. B. Myoepithelial cell density (cell/mm²) was quantified in periductal regions is shown for normal breast, pure $\mathrm{DCIS}$, and synchronous DCIS samples. C. The frequency of Ki67 (top) and pS6 (bottom) positivity is compared between groups as in B. D. Illustration of workflow for quantifying myoepithelial thickness and continuity. E. Boxplot showing myoepithelial integrity (percent coverage $x$ average thickness) for normal tissue and patients with pure or synchronous DCIS. F. Workflow schematic for pixel-based clustering of myoepithelial phenotype. G. Heatmap showing frequency and average marker expression for 7 myoepithelial pixel clusters (mc) with a bar plot (left) of mc abundance out of total identified myoepithelium in the cohort. H. Top. Pseudo-colored image illustrating the spatial distribution of myoepithelial pixel clusters defined in $G$ for a pure (left) and synchronous (right) DCIS tumor, scale bars $=50 \mu \mathrm{m}$. Middle. Magnified periductal region with mcECAD (pink arrows), mcCK5 (orange arrow), and mcVIM (yellow arrow) areas denoted. Bottom. Coregistered color overlays showing variations in coexpression of SMA, ECAD, CK5, and VIM corresponding to pixel cluster assignments, scale bars $=50 \mu \mathrm{m}$. I. Area plots comparing the frequency of each myoep cluster across normal breast, pure, and synchronous DCIS.

258 Due to their thin, elongated, and non-spherical cell bodies, myoepithelial cells are 259 inherently challenging to profile with classical nuclear-based segmentation approaches 260 which have been optimized for more conventional, ovoid cell shapes. Consequently, 261 outlines for myoepithelial cells predicted by these methods often extend significantly 262 beyond the true cellular border to erroneously include pixels from neighboring epithelial 263 and stromal cells. These errors propagate in downstream cell clustering analyses to 264 result in inaccurate phenotypic descriptions that are biased by what proteins are expressed by closely approximated neighboring cells.

To avoid this pitfall, we created a new computational approach that assigns phenotypes at the level of single pixels, rather than for whole cells (Figure 4F, see Myoepithelial Pixel Clustering Analysis in Methods). This strategy yielded 7 distinct, $\mathrm{SMA}^{+}$myoepithelial pixel clusters (mc) defined by coexpression of PanCK, ECAD, CK7, CK5, VIM, or CD44, with SMA (Figure 4G). Mapping these pixel clusters back onto the

271 original images revealed that multiple expressional states can exist along the perimeter 272 of a single duct, from $\mathrm{ECAD}^{+}$and $\mathrm{CK}^{+}$expression states often observed with apical 273 preference (Figure $\mathrm{H}$, pink and green arrows), and more mesenchymal states that 274 exhibited a basal preference (e.g., $\mathrm{VIM}^{+}, \mathrm{CD}_{4} 4^{+}$, yellow arrows). Notably, this analysis 275 also revealed a transition from a more luminal-like state in normal samples to a more 276 mesenchymal-like state in synchronous DCIS that aligned with analogous shifts in tumor 277 cell differentiation and function (Figure 4I).

279 Fibroblast transition and collagen architecture remodeling during DCIS 280 tumorigenesis and progression 
281 In light of previous studies revealing a functional and structural interdependence between myoepithelium and the surrounding stroma (Jones et al., 2003; Morsing et al., 2020), we next sought to determine if the progressive loss of myoepithelial integrity observed here correlated with changes in fibroblast function and extracellular matrix remodeling (ECM). Single cell clustering revealed four fibroblast populations that included normal (CD36 high), resting (VIM-only), myofibroblast $\left(\mathrm{SMA}^{+}\right)$, and $\mathrm{CAF}\left(\mathrm{FAP}^{+}\right)$subsets (Figure $\left.5 \mathrm{~A}\right)$. No significant differences in stromal cell density between progression groups were identified when treating fibroblasts as a single cell population (Figure 5B). However, on comparing the frequency of fibroblast subsets in normal tissue and DCIS, CAFs were found to significantly increase across tumor progression as resting fibroblasts decreased (Figure $5 \mathrm{C}$ ), with pure DCIS tumors having a heterogeneous mixture of these two states (Figure 292 5D, normal fibroblasts with light blue arrows, CAFs with dark blue arrows). A corresponding increase in $\mathrm{Ki} 67^{+}$fibroblasts suggests that this shift in identity is driven in part by CAF proliferation (Figure $5 E$ ), which is accompanied by an increase in protein translation (high pS6). We confirmed this relationship by comparing the CAF frequency in samples with high and low pS6 and Ki67 (Figure S4A-B).

Given these findings, and that dense fibrillar collagen often appeared to be juxtaposed with $\mathrm{pS6}^{+}$fibroblasts in progressed tumors (Figure 5F, orange arrows), we next sought to determine how collagen remodeling was related to CAF location, frequency, and phenotype. To achieve this, we developed new computational tools for collagen morphometrics that were used to determine the shape, length, and density of individual fibers (Figure 5G, see Collagen Morphometrics in Methods). These analyses revealed that DCIS and IBC tumors had higher collagen density and longer fiber length compared to normal breast (Figure $5 \mathrm{H}$ ), suggesting that collagen deposition and fibrillar remodeling were coordinated with the phenotypic shift to CAFs. Indeed, direct comparison of collagen density and collagen-positive area to the density of CAFs and myofibroblasts in the stroma revealed a strong correlation (Figure 5I). Furthermore, pS6 ${ }^{+}$ fibroblasts were also enriched in these collagen and CAF-dense tumors. Together these data suggest a direct relationship between CAF activation and collagen deposition and 310 remodeling. 
bioRxiv preprint doi: https://doi.org/10.1101/2021.01.05.425362; this version posted January 6, 2021. The copyright holder for this preprint (which was not certified by peer review) is the author/funder, who has granted bioRxiv a license to display the preprint in perpetuity. It is made available under aCC-BY-NC-ND 4.0 International license.
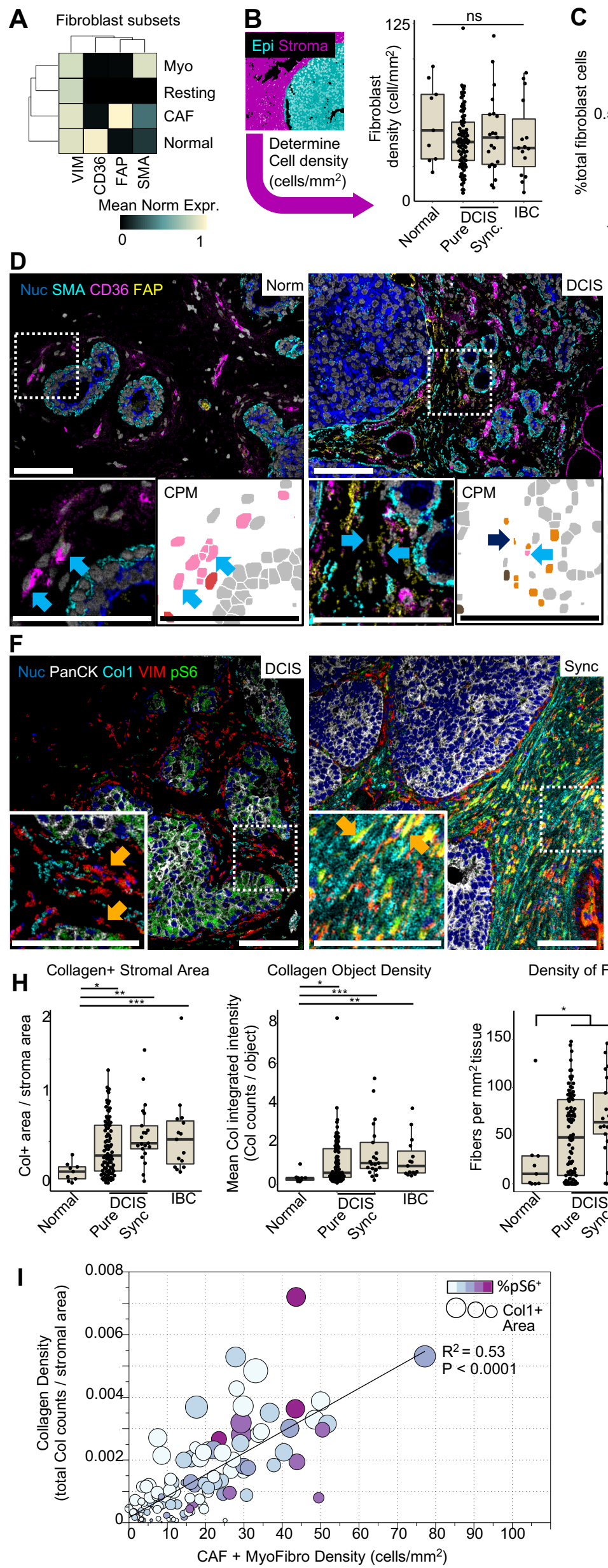
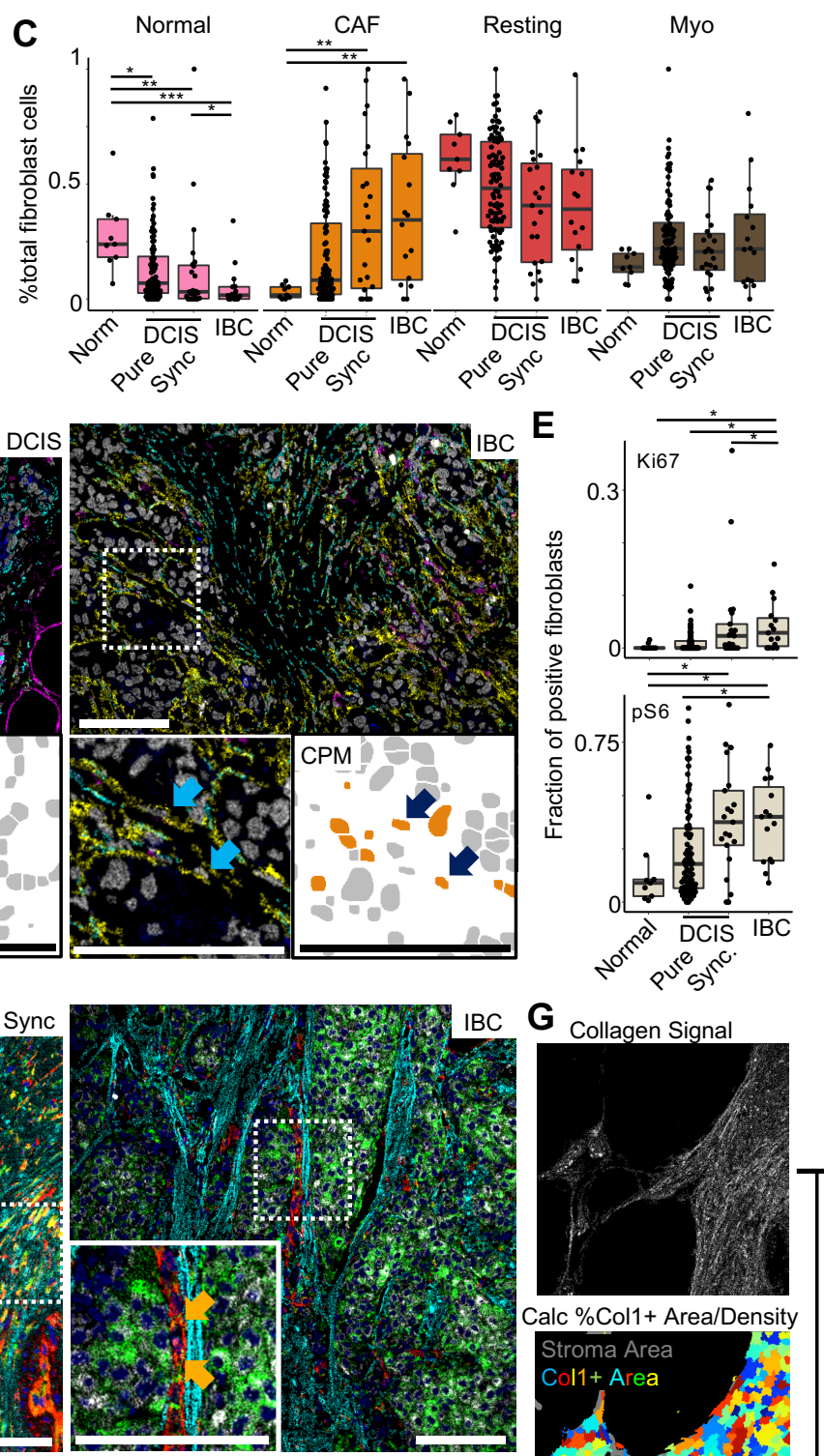

Density of Fibers

Mean Fiber Area
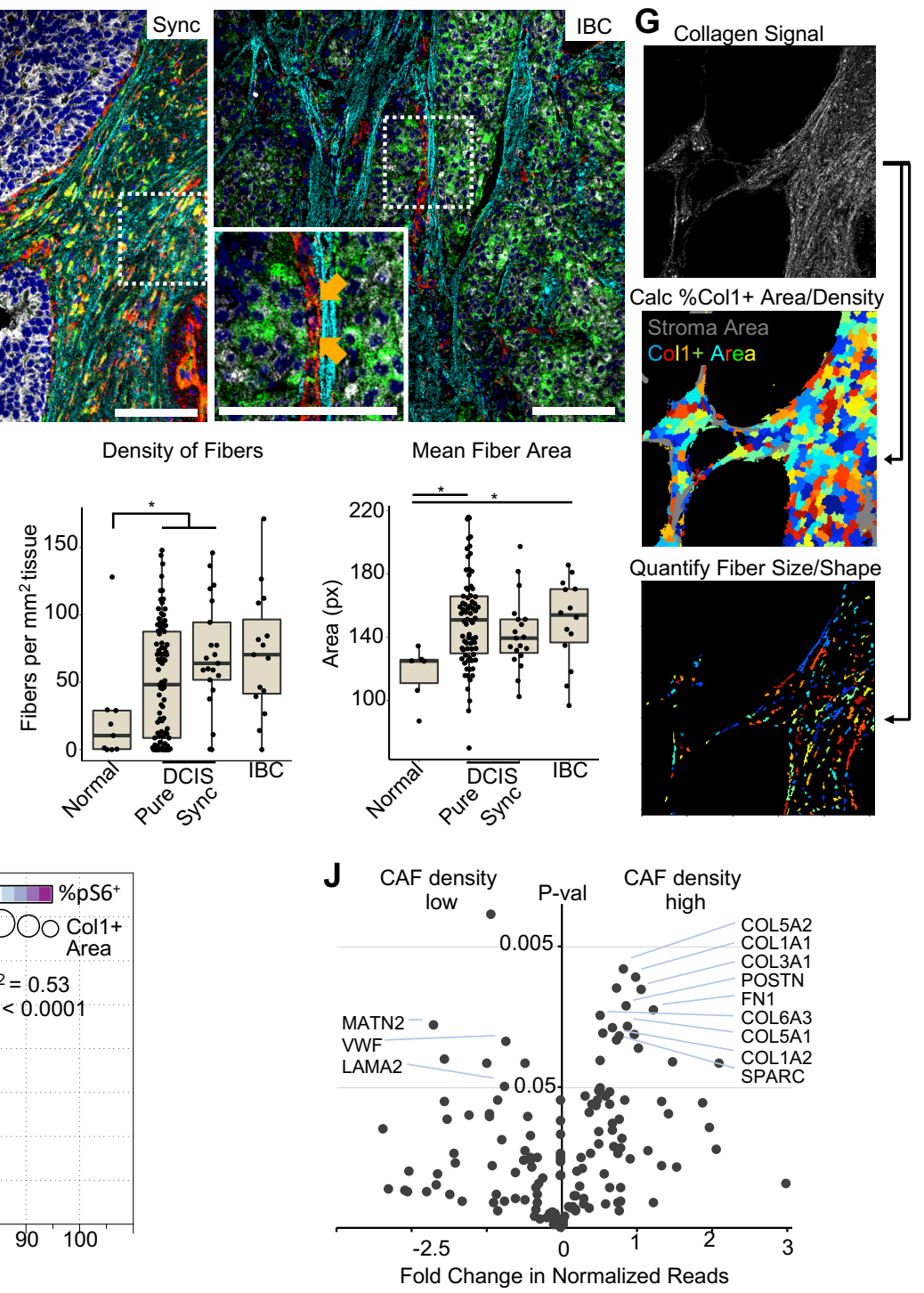
Figure 5. Fibroblast transition and collagen architecture remodeling during $\mathrm{DCIS}$ tumorigenesis and progression

A. Heatmap showing normalized marker expression for four fibroblast cell subsets: myofibroblasts (Myo), resting fibroblasts (Resting), cancerassociated fibroblasts (CAFs) and normal fibroblasts (Normal). B. Left. Example epithelial (cyan) and stromal (magenta) masks used to quantify stromal fibroblast density. Right. Boxplot of fibroblast density between tumor progression groups. C. Boxplots of fibroblast subset frequency across tumor progression groups. D. Representative MIBI image overlays showing normal, pure DCIS, and sync DCIS tumors with fibroblast markers. Zoomed insets (left) have paired cell phenotype maps (CPM, right) colored by fibroblast identity as in C, scale bars $=100 \mu m$. E. The frequency of Ki67 and pS6 positivity in fibroblasts is shown across progression groups. F. Representative MIBI image overlays showing $\mathrm{VIM}^{+}$fibroblasts (red) with varying levels of pS6 expression (green) and nearby collagen 1 (Col1, cyan) deposition, scale bars $=100 \mu \mathrm{m}$. G. Schematic showing the quantitation of MIBI collagen signal to identify \%collagen+ stromal area, collagen density, and collagen fiber morphometrics. H. Collagen+ stromal area, collagen density, collagen fiber density (fibers $/ \mathrm{mm}^{2}$ ) and fiber area are quantified across tumor progression groups. I. Scatterplot comparing summed density of CAFs and myofibroblasts versus collagen density. Size and color of points are proportional to collagenized area and fibroblast pS6 positivity, respectively. J. Volcano plot of ECM-related gene expression for the top and bottom CAF-enriched DCIS tumors.

311 Finally, to identify which specific collagen isoforms correlate with this activity and

312 to determine if additional ECM proteins are involved, we compared ECM transcript levels

313 in stroma of CAF-high- and low-density tumors using LCM RNAseq. We found the

314 majority of collagen species were upregulated in CAF-high tumors with COL5A2 and

315 COL1A1 being the most significant of these, consistent with MIBI-TOF quantitation of

316 COL1A1 protein (Figure 5J). In addition, CAF-dense tumors showed increased deposition

317 of fibronectin (FN1), SPARC and periostin (POSTN), indicative of CAF-remodeling and a

318 shift towards a pro-invasive stroma (Barth et al., 2005; Malanchi et al., 2012).

Characterizing the preinvasive immune microenvironment and its compartmental evolution throughout progression

322 Having identified coordinated shifts in tumor differentiation, myoepithelial integrity, and 323 fibroblast function, we next sought to understand how immune composition changed with 324 disease progression. We found monocytes, mast cells, and $\mathrm{HLA}^{-\mathrm{DR}^{+}}$antigen presenting 325 cells (APCs) to be the most abundant immune cells in pure DCIS (Figure 6A). Immune 326 cells were typically found in the stroma and were occasionally embedded in ducts (Figure 327 B, orange arrow). To quantify the spatial distribution of immune cells in these 328 compartments, we interrogated cell density in epithelial and stromal mask regions (Figure $3296 \mathrm{C}$ ). This analysis identified a clear stromal preference when treating immune cells as a 330 single population (Figure 6D, S5A). To understand if this preference remained valid when 331 considering specific subsets of lymphoid and myeloid cells, we compared the local 332 frequency within stromal and ductal regions for each cell type. CD4 ${ }^{+} \mathrm{T}$ cells, B cells, 333 monocytes, APCs and mast cells all demonstrated a statistically significant stromal 
334 preference, while macrophages were significantly enriched in ductal regions (Figure 335 6E). Interestingly, differential enrichment of $\mathrm{CD}^{+}$and $\mathrm{CD}^{+} \mathrm{T}$ cells resulted in a 336 CD4/CD8 ratio that skewed towards $\mathrm{CD}^{+} \mathrm{T}$ cells in ducts and $\mathrm{CD}^{+} \mathrm{T}$ cells in stroma 337 (Figure 6F).

We next investigated how immune cell prevalence and spatial enrichment evolves with transition from in situ tumorigenesis to invasive disease by comparing pure DCIS with synchronous lesions and IBC. Immune cell density was significantly increased in synchronous lesions compared to all other groups (Figure 6G). Notably, this increase in immune infiltrate was present in both the stroma and ducts of these lesions (Figure $6 \mathrm{H}$ ), suggesting a coordinated influx into the ducts during increased stromal immune infiltration. By comparing the cell density for each immune cell subset with respect to disease stage, we observed an increase in effector myeloid cells (Macs, APC) in pure DCIS compared to normal breast (Figure 6I). Importantly, this also revealed the increase in immune infiltrate in synchronous tumors to be driven primarily by an influx of $B$ and $T$ lymphocytes (Figure 6I, S5B), resulting in an immune microenvironment more skewed towards lymphocytes (Figure 6J). Subsequently, both T cell frequency and myeloid to lymphoid ratio in IBC tumors return to values similar to pure DCIS.

In order to better understand how this feature and other immune programs were spatially organized, we applied a K-means clustering approach to identify distinct cellular neighborhoods (CNs), where a $\mathrm{CN}$ is defined by a set of cell types found to spatially cooccur across the cohort (Figure 6K, see Protein and Cellular Spatial Enrichment Analyses in Methods). Through this approach, we identified $10 \mathrm{CNs}$ that we categorized as being lymphocyte-enriched (LyE1, LyE2), myeloid-enriched (MyE), endothelial-associated (EA), fibroblast-associated (FA), myoepithelial-associated (MA), tumor-interface (TI), and tumor-enriched (TE1-3, Figure 6L-N).

Interestingly, single cell expression of functional markers was found to be 360 correlated with $\mathrm{CN}$, even though these parameters were not included in the K-means 361 neighborhood assignment analysis. For example, HIF1 $\alpha$ and MMP9 expressing cells 362 were enriched in MyE, while the frequency of $\mathrm{pS6}^{+}$cells was highest in LyE1 (Figure $3636 \mathrm{~L})$. Macrophages were a constituent of numerous CNs and showed functional state 364 distinction based on neighborhood association, including increased PDL1 expression 


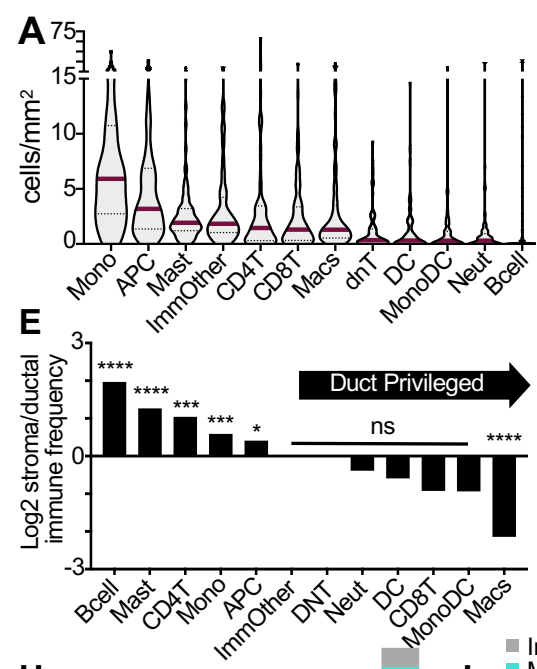

H

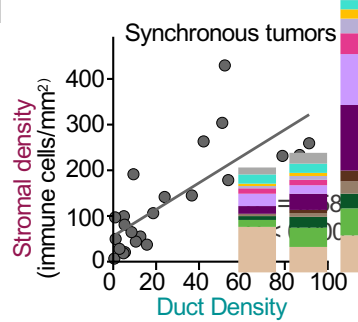

$\mathbf{L}$

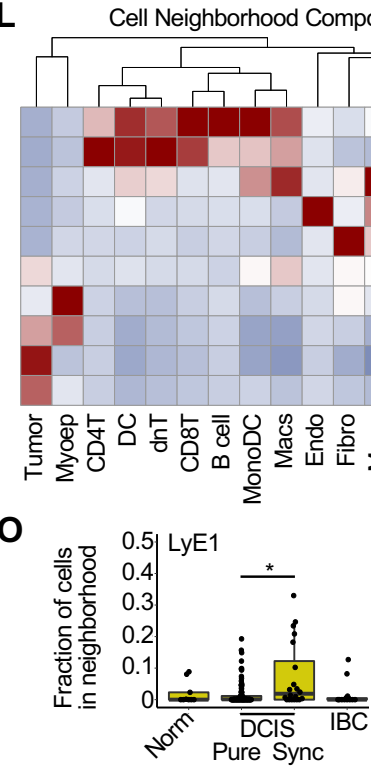

mam

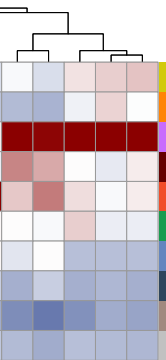

B
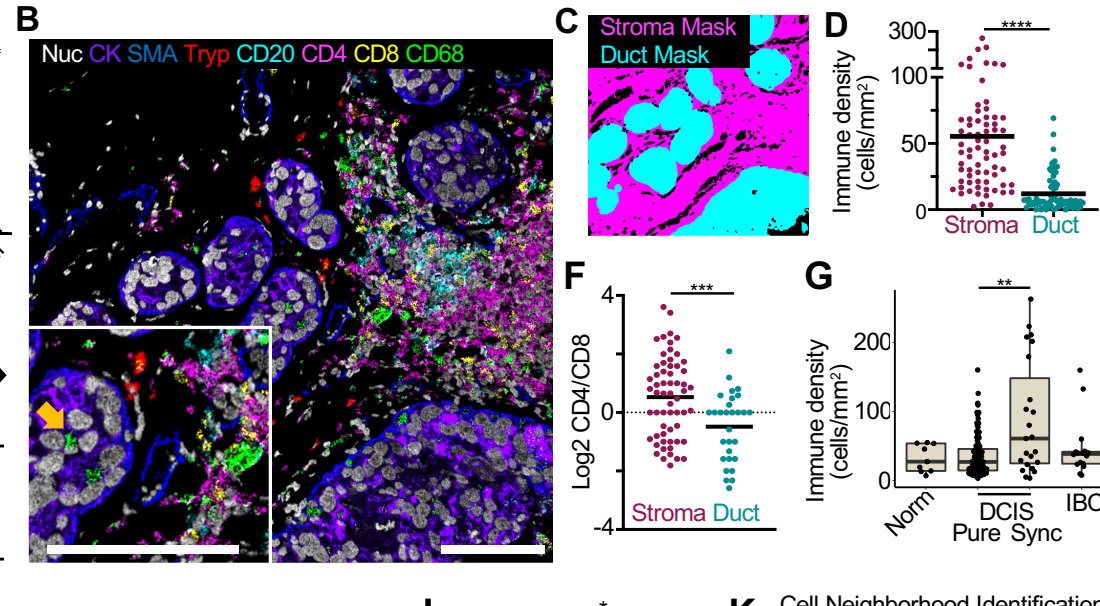

$F_{4+\infty+t} \quad \mathbf{G}$
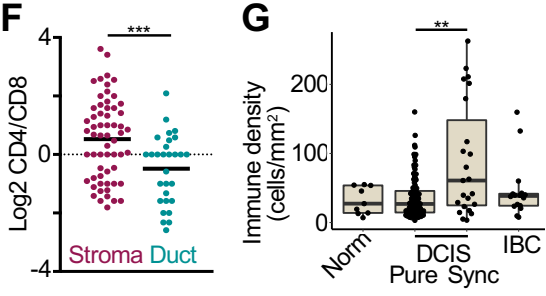

ImmOth

Mast Neut B cell BD8T CD4T - MonoDC
$-\mathrm{DC}$ Macs APC Mono
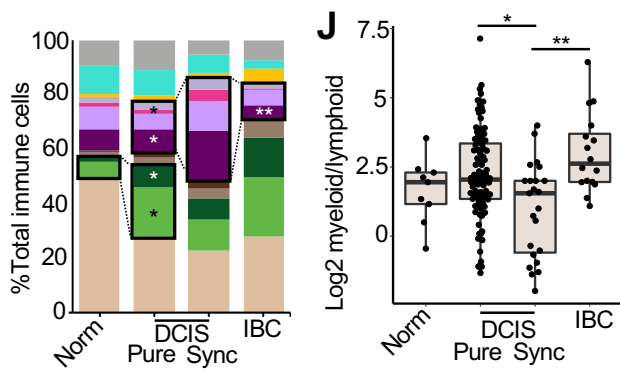

K

Cell Neighborhood Identification

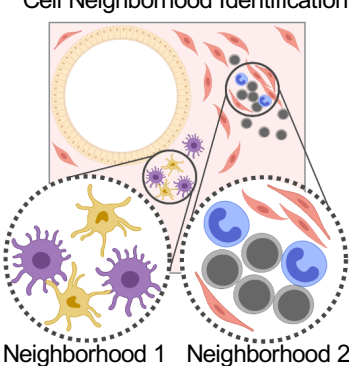

Neighböhood 1 Neighbörhood 2
M

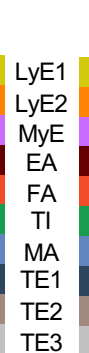

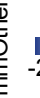

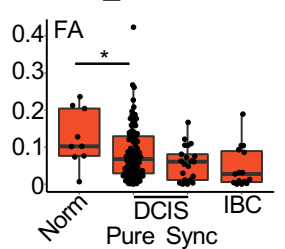

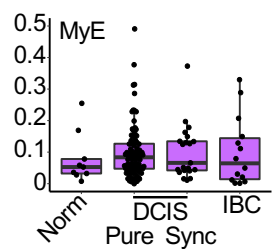

$\mathbf{N}$

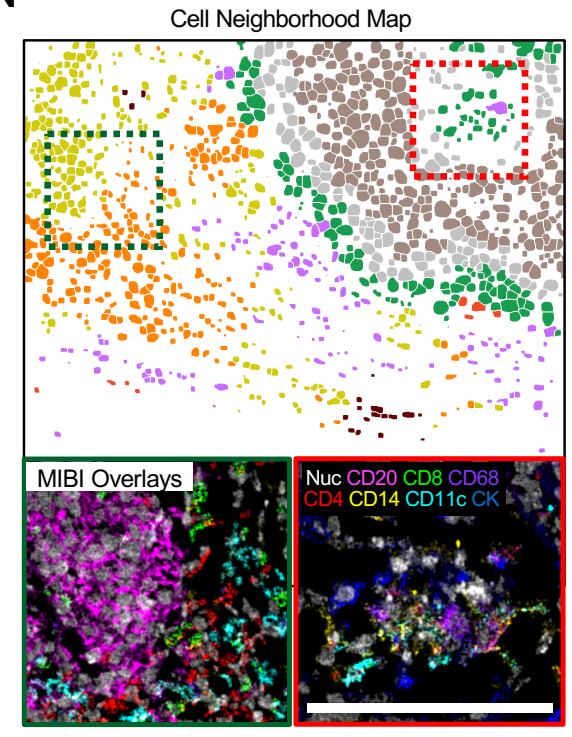

Figure 6. Characterizing the preinvasive immune microenvironment and its compartmental evolution throughout progression A. Violin plot examining immune cell density in pure DCIS, ranked by median density per patient. B. Representative MIBI image overlay of a pure DCIS tumor with major immune cell type markers, inset and arrow highlighting intraductal immune phenotypes. C. Mask overlay showing delineation of stroma and duct regions in $B$, scale bars $=100 \mu \mathrm{m}$. D. Scatterplot comparing immune cell density between the stroma and duct compartments per patient. E. Column plot showing the ratio (Log2) of immune cell type frequency between stroma and ductal compartments, ranked from high (stromal preference) to low (duct preference). Asterisks denote significance comparing compartment frequency of a given cell type across all pure DCIS patients. F. Log2 ratio of $\mathrm{CD}^{+}$to $\mathrm{CD} 8^{+} \mathrm{T}$ cells is displayed per patient for the stroma and duct compartments. G. Whole image immune density is compared across tumor progression groups. H. Scatterplot comparing stromal and ductal immune density per patient in synchronous tumors. I. Area plot showing the change in immune subset frequency across progression groups. Effector-myeloid cell subsets are boxed and compared between normal breast and pure DCIS tumors; asterisks denote significant differences in frequency. Lymphocyte subsets are boxed and compared between pure DCIS, synchronous DCIS, and IBC, asterisks denote significance vs the synchronous group. J. Boxplots showing the log2 ratio of myeloid to lymphoid cells in tumor progression groups. K. Illustration depicting different spatially-enriched cellular neighborhoods. L. Heatmap showing z-score normalized cell type frequency for each cellular neighborhood: Iymphocyte-enriched (LyE1, LyE2), myeloid-enriched (MyE), endothelial-associated (EA), fibroblast-associated (FA), tumor-interface (TI), myoepithelial-associated (MA), and tumor-enriched (TE1-3). M. Heatmaps showing Z-score normalized mean expression for functional markers in each cellular neighborhood. N. Top. Cell neighborhood map showing the spatial localization of distinct neighborhoods, denoted by color as in M. Bottom. Color overlays for lymphocyte-enriched (green dotted line) or tumor-interface (red dotted line), scale bar $=100 \mu \mathrm{m}$. O. Boxplot showing frequency of cells assigned to LyE1 (yellow), Fibroblast-associated (Red) and MyE (purple) cell neighborhoods across tumor progression groups. 
365 within LyE neighborhoods, in addition to pS6 (Figure S5C-D). Notably, the LyE1

366 neighborhood was also enriched for $T$ and B cells, consistent with tertiary lymphoid 367 structure formation (see Figure $6 \mathrm{~N}$, bottom left). In line with the trends observed for $\mathrm{T}$ cell 368 infiltrates, we found the frequency of cells belonging to LyE1 to be increased in 369 synchronous lesions (Figure 60). Taken together, these findings indicate that early 370 stromal invasion in synchronous tumors triggers an influx of T cells and formation of TLS 371 structures. We find that by IBC, however, the tumor immune microenvironment has 372 reverted to a myeloid-skewed, immunosuppressed state with diminished $\mathrm{T}$ cell 373 presence.

Identifying DCIS features correlated with recurrence outcomes

376 Having extensively quantified the multi-compartmental cellular and structural elements of 377 DCIS tumors, we leveraged these data to identify features associated with the risk of 378 recurrence following primary DCIS resection. We selectively examined these features in 379 diagnostic tissue procured at the time of initial presentation in two sets of patients. The 380 first set, referred to as "case", consisted of 31 patients who had a recurrence (DCIS or 381 IBC) within 2-15 years of being treated for newly diagnosed pure DCIS. The second set, 382 referred to as "control", consisted of 47 patients with pure DCIS that did not recur within $38311+$ years.

$384 \quad$ Using these outcome groups and 1,093 phenotypic, functional, spatial, and 385 morphologic features extracted from our MIBI-TOF analyses (Table S3), we trained two random forest classifier models. The first was an all-recurrence model for predicting 387 which patients would have a recurrence of DCIS or IBC. The second was an invasive 388 recurrence model for predicting IBC recurrence exclusively (Figure 7A). Low observation and overly correlated features were removed from the dataset and the patient population was randomly split 80/20 to training and test groups. We evaluated classifier accuracy in 391 the withheld test set, where the all-recurrence and invasive models achieved an AUC of $3920.79(\mathrm{Cl} 0.51: 1)$ and $0.83(\mathrm{Cl} 0.59: 1)$, respectively (Figure $7 \mathrm{~B})$. When stratifying patients 393 by their predicted labels, we found a significant difference in recurrence probability over 394 time (Fig. 7C, Figure S6A), with no recurrence events in the patients predicted by the 395 invasive model to be non-progressors. Although sample size precluded us from being 
bioRxiv preprint doi: https://doi.org/10.1101/2021.01.05.425362; this version posted January 6, 2021. The copyright holder for this preprint (which was not certified by peer review) is the author/funder, who has granted bioRxiv a license to display the preprint in perpetuity. It is made available under aCC-BY-NC-ND 4.0 International license.

A 1: Separate primary DCIS outcome groups

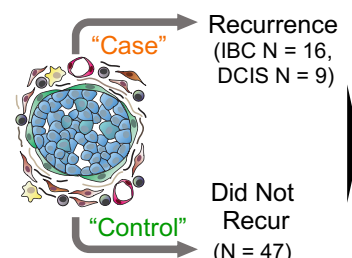

$(\mathrm{N}=47)$

B $\rightarrow$ Invasive Recurrence Model

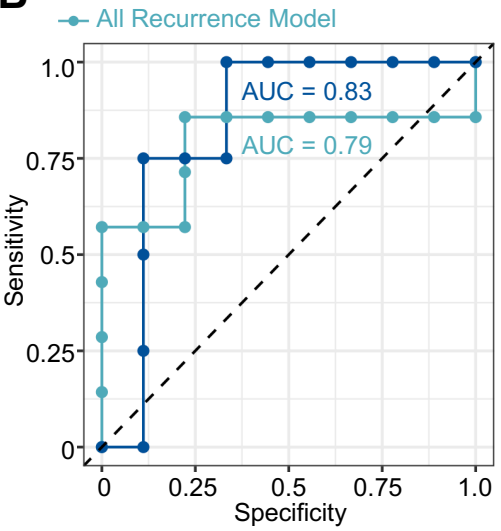

Predicted Progression
Free Survival $(p=0.034)$
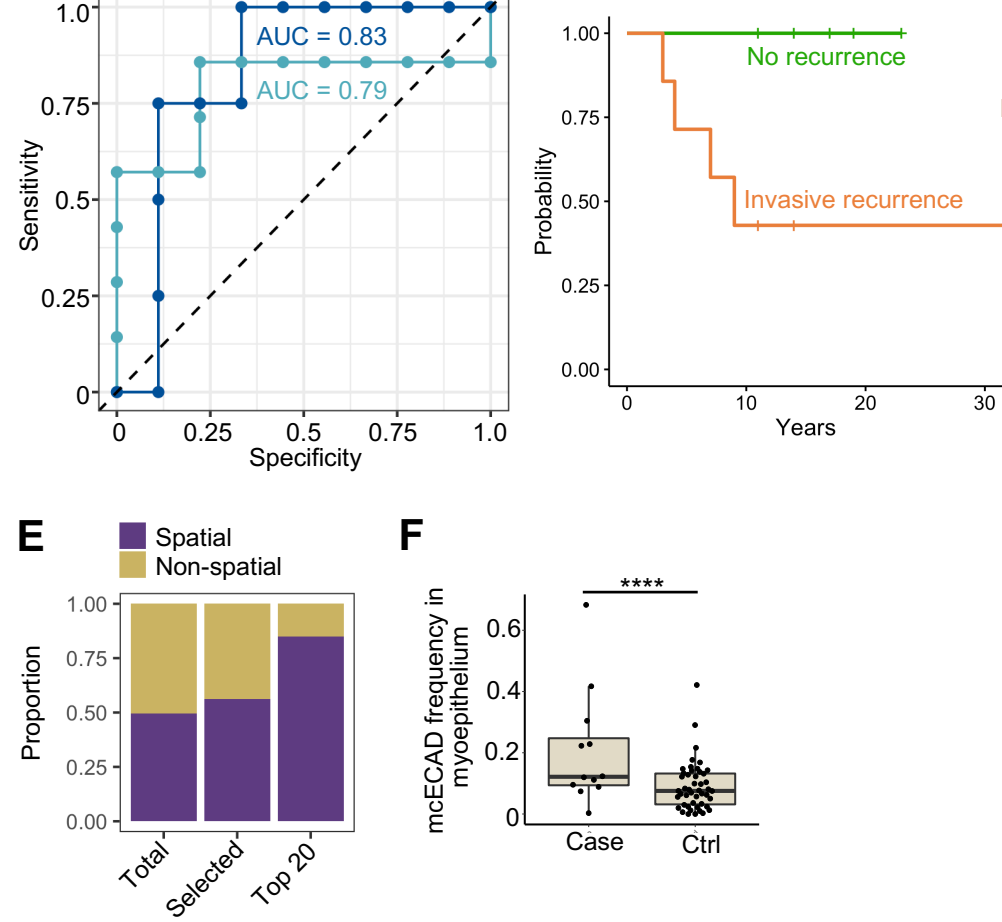

$F$

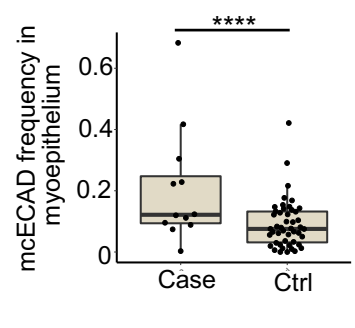

Total $=1093 \mathrm{MIBI}$ Features
3: Split patients into training and test groups

4: Train and evaluate classifier

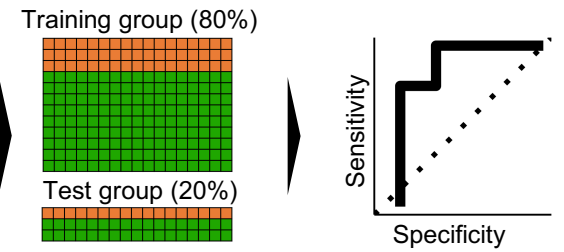

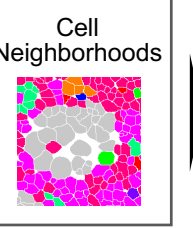

D

Myoepithelium mcECAD frequencyAPC to endothelium distance. Myoepithelium to APC distance $E C A D^{+}$frequency in immune cells StDev of collagen fiber-fiber alignment. Density of $\mathrm{PanCK}^{+} \mathrm{VIM}^{+}$cells on stroma edge$\mathrm{dnT}$ cell to CD8T cell distance

Monocyte to monocyte distance. Macrophage to immune other distanceMast cell to macrophage distance Epithelial density of macrophages Myoepithelial cell $\mathrm{CK}^{+}$frequency Percent of duct covered by myoepithelium Neighborhood TE3 fractionStromal density of mast cells Max intensity of collagen in stromaMacrophage to macrophage distanceAPC to CD8 T distanceVariation in collagen fiber areadnT cell GLUT1+ frequency (enrichment: case / control) 0

Gini Importance

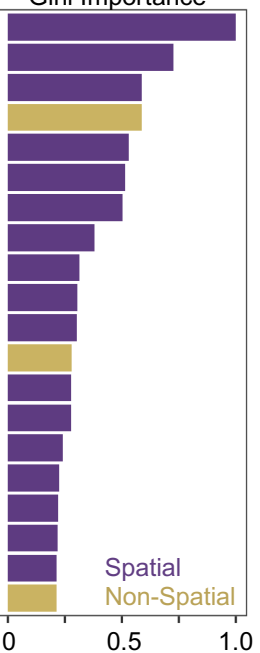

\section{Figure 7. Identifying DCIS features correlated with recurrence outcomes}

A. Schematic illustrating the different outcome groups of primary DCIS including "cases" that recurred either as IBC or DCIS, and "controls" with no recurrence in $>11 \mathrm{yr}$ follow-up. 1,093 MIBI features of numerous tumor metrics were used to train a random forest classifier to differentiate case and control samples. Classifier specificity was then tested on a withheld $20 \%$ of patients. B. AUC plot showing classifier sensitivity and specificity. C. Predicted survival of patients identified in the test set of the invasive-recurrence model as case or control. D. MIBI features with top classifier importance for the IBC recurrence model are shown, ranked by Gini importance. Features are colored based on enrichment either in cases (orange) or controls (green), importance bars are colored based on the feature utilizing spatial information (purple) or not (gold). E. The distribution of spatial vs non-spatial features are shown for all features identified (total), those used by the model (selected), and those in the top 20 most important features (top 20). F. Boxplot showing the frequency of the mcECAD myoepithelial phenotype between invasive cases and controls. 
To understand the biology being leveraged by this classifier to accurately discriminate pre-invasive from indolent DCIS tumors, we ranked the top 20 features based on Gini importance. These features primarily consisted of metrics related to the phenotype of myoepithelium, the structure of collagen fibers in the extracellular matrix, and the spatial distribution of multiple immune cell subsets (Figure 7C). Notably, spatial metrics describing cell densities, cell neighborhoods, pairwise cell distances, collagen structure, and multiplexed subcellular features were overrepresented and accounted for 17 of the top 20 metrics in the invasive model (Figure 7D, Table S3). Immune cell metrics comprised about half of these and were myeloid skewed (Figure S6D, with 9 relating specifically to myeloid subsets and 3 to lymphoid subsets. Similarly, enrichment for spatial metrics related to myoepithelium, collagen, and myeloid cells were observed in

412 the all-recurrence model as well (Figure S6D-F). Stromal density of $\mathrm{PanCK}^{+} \mathrm{VIM}^{+}$cells ranked in the top 20 features. These cells were rare (median of 0 in case and controls) and on manual inspection appeared to represent fibroblasts where PanCK expression from closely neighboring epithelial cells was misassigned. Interestingly, both models identified pixel-level, $\mathrm{ECAD}^{+}$myoepithelial expression as the most predictive metric (mcECAD, see Figure 4). When comparing case and control samples, we found the frequency of this feature to be significantly different between these outcome groups, independent of the classifier model, and to be readily identifiable on targeted inspection of the original imaging data $(p<0.001$, Figure 7F).

\section{Discussion}

424 Here, we report the first multicompartmental atlas of the single cell composition and 425 structure of DCIS. The central focus of this study was to characterize the changes 426 undergone with progression to IBC where tumor cells breach the duct to invade the 427 surrounding stroma. Previous work examining BC progression have attempted to 428 attribute this transition either to tumor-intrinsic factors or to specific features of stromal 429 cells in the surrounding TME. By simultaneously mapping both tumor and stromal cell 430 identity and function in intact human tissue, we sought to treat the DCIS TME as a single 
431

432

433

434

435

436

437

438

439

440

441

442

443

444

445

446

447

448

449

450

451

452

453

454

455

456

457

458

459

460

461

ecosystem where progression to invasive disease depends on the spatial distribution and function of multiple cell types, rather than on any single cell subset.

Meeting this goal required first assembling a large, well-annotated, and diversified pool of human DCIS tissue: the RAHBT cohort. This effort was motivated in part by the success of similar work investigating invasive disease (i.e. METABRIC) that have provided deep insights into breast tumor composition and have served as authoritative resources in breast cancer research (Curtis et al., 2012). To achieve this, the Breast PreCancer Atlas constructed a unique set of archival human surgical resections that captured the full spectrum of breast cancer progression, from normal tissue, to pure DCIS and IBC. Assembling all of these cases into TMAs has enabled a one-of-a-kind workflow for multiomics analyses where genomic, transcriptomic, and proteomic techniques are performed not only on the same samples, but on coregistered serial sections of the same local region of tissue.

Here, we describe the first major analysis of the RAHBT cohort where high dimensional imaging was used to characterize BC progression. We used MIBI-TOF for subcellular imaging of 140 tumor and normal breast samples using a 37-marker staining panel (122 and 23 samples from RAHBT and Stanford cohorts, respectively). Tumor cell differentiation and function were found to transition along a continuum from pronounced luminal features in normal breast to a more undifferentiated, cytokeratin-low state in invasive disease that had increased mesenchymal features. This shift was accompanied by an upregulation of HIF1 $\alpha$, MMP9, and IDO in tumor cells, which have been shown to directly elicit EMT, promote invasion, and drive immune tolerance, respectively (Kolijn et al., 2018; Lin et al., 2011; Peng et al., 2018; Zhang et al., 2015, 2019). With transition to DCIS, the frequency of an E-cadherin-high myoepithelial phenotype that predominated normal breast tissue decreased, as a more mesenchymal, CD44- and VIM-high state increased. Interestingly, no difference in myoepithelial cell density or structural integrity was found when comparing DCIS in pure and synchronous lesions. Given that the invasive and in situ components of synchronous tumors are closely related on a genomic level (Ak et al., 2018; Kim et al., 2015; Newburger et al., 2013) these findings suggest that transition to invasion disease is regulated at least in part by the local microenvironment. 
These epithelial changes were accompanied by a stromal transition towards higher

463

464

465

466

467

468

469

470

471

472

473

474

475

476

477

478

479

480

481

482

483

484

485

486

487

488

489

490

491

492 numbers of activated, proliferating CAFs and densely aligned fibrillar collagen (Conklin et al., 2011; Esbona et al., 2018). Although the total immune density was comparable to normal breast tissue, DCIS tumors exhibited a shift from a monocyte-predominant environment to one enriched for APCs and intraductal macrophages. In line with recent findings by other groups (Alcazar et al., 2017; Kim et al., 2020) synchronous DCIS/IBC tumors were marked by a stromal spike in T and B cells and formation of tertiary lymphoid structures. This feature distinguishes them from the myeloid-skewed IBC samples profiled in this study. Taken together, these findings support a model for breast cancer progression where invasive disease occurs through multiple coordinated, dynamic interactions of the surrounding stroma, myoepithelium, and tumor.

Given the urgent need to better stratify DCIS patients based on risk of progression, we tested to see if these spatial and phenotypic features could be used to predict IBC recurrence based exclusively on diagnostic DCIS tissue. Using 1,093 features, we trained a random forest classifier model for identifying patients that would later progress to IBC that achieved an AUC of 0.83 on withheld test samples. Although the performance was impressive, certain caveats should be taken into account when considering how generalized this model might be. Given the complexity of breast cancer subtypes and the impact of patient demographics on outcome (Alaeikhanehshir et al., 2020; Liu et al., 2019), the sample size in this study may not have been sufficient to fully account for the confounding effects of these variables. Lastly, since all patients in the RAHBT cohort received one or more therapeutic interventions, the features leveraged by this model to identify non-progressors might not be valid when applied to patient populations where therapy is omitted.

With these considerations in mind however, these results do offer three compelling and overarching insights. First, spatial metrics relating phenotype to structure and morphology were significantly over-represented relative to non-spatial metrics, accounting for almost $85 \%$ of the top 20 features identified by the classifier model. Second, the most influential features were primarily related to the stroma rather than the tumor cells themselves. This included a previously unreported E-cadherin high 
493 myoepithelial phenotype as well as collagen fiber size and alignment with respect to the duct. Third, high ranking immune features more often related to myeloid than to lymphoid

495 subsets, particularly those in close proximity with myoepithelium or residing inside the 496 duct. This skewing underscores the need to better understand how macrophages promote TME immune suppression, tumor proliferation, and local invasion (Esbona et al., 2018; Goswami et al., 2005; Linde et al., 2018; Ruffell et al., 2012).

Taken together, this study offers a comprehensive, multi-compartmental atlas of preinvasive breast cancer that illustrates the full continuum of tissue structure and

501 function starting from a homeostatic state in normal breast through in situ and invasive 502 disease. Combining this comprehensive data set with extensive patient follow-up has 503 enabled identification of tumor features that are associated with DCIS recurrence and 504 offers a framework for exciting follow-on efforts. With this in mind, we are actively planning 505 a larger study that will further evaluate the biological significance of spatial features 506 relating to myoepithelium, collagen, and myeloid cells and to determine if they can be used to prospectively risk stratify patients with a new DCIS diagnosis.

\section{Methods}

\section{Patient Cohort}

513 We utilized a retrospective study cohort of patients from the Washington University 514 Resource of Archival Tissue (RAHBT) that contained two outcome groups: controls 515 ("Ctrl") composed of patients with DCIS who had no recurrence and cases ("Case") 516 composed of patients with DCIS who had either a DCIS or an IBC recurrence. For each 517 case, we matched two controls who remained free from recurrent lesions, based on age 518 at diagnosis (+/- 5 years), and type of definitive surgery (mastectomy or lumpectomy). For 519 each DCIS diagnosis we retrieved primary and recurrent tumor slides and blocks for 520 pathology review, secured a whole slide image of each sample, marked for TMA cores, 521 and generated TMA blocks with $841.5 \mathrm{~mm}$ cores, including additional tonsil and normal 522 breast controls. 
Supplemental table 1 summarizes the data for the cases in the cohort. Median age at diagnosis was 54, year of diagnosis was 1986 to 2017, and time to recurrence with was 8.8 years for invasive lesions, and 5.3 years for premalignant lesions. For women in

526 the cohort with no recurrence, follow up extended to 132 months, on average. Treatment

527 of initial DCIS ranged from lumpectomy with radiation (approximately half of cases), and

528 lumpectomy with no radiation (20\%) and mastectomy with no radiation for $30 \%$. The 529 RAHBT cohort is composed of African American women (26\%) and white women (74\%). 530 We also profiled a supplemental cohort of patients from the Stanford Hospital with 531 synchronous ("Sync") DCIS and IBC tumors from 2007-2009. A 216-core TMA block was 532 generated with $1 \mathrm{~mm}$ tumor cores, with additional tissue controls.

$5 \mu \mathrm{m}$ serial sections of each TMA slide were cut onto glass slides for hematoxylin and eosin (H\&E) staining, onto laser-capture slides for LCM-RNAseq (SMART-3SEQ) and cut onto gold- and tantalum-sputtered slides for MIBI-TOF imaging. H\&E slides were inspected by a breast cancer pathologist to address DCIS purity and demarcate regions of DCIS to guide MIBI imaging and laser dissection of epithelial and stromal area. The Stanford Hospital cohort was without paired LCM-RNAseq analysis.

\section{Antibody Preparation}

541 Antibodies were conjugated to isotopic metal reporters as described previously (Keren et 542 al., 2018; McCaffrey et al., 2020). Following conjugation antibodies were diluted in Candor 543 PBS Antibody Stabilization solution (Candor Bioscience). Antibodies were either stored

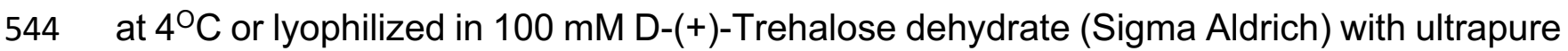
545 distilled $\mathrm{H} 2 \mathrm{O}$ for storage at $-200 \mathrm{C}$. Prior to staining, lyophilized antibodies were reconstituted in a buffer of Tris (Thermo Fisher Scientific), sodium azide (Sigma Aldrich),

547 ultrapure water (Thermo Fisher Scientific), and antibody stabilizer (Candor Bioscience) to 548 a concentration of $0.05 \mathrm{mg} / \mathrm{mL}$. Some metal-conjugated antibodies in this study were 549 used as secondary antibodies, targeting hapten groups on hapten-conjugated primary 550 antibodies, this included the pairs PDL1-Biotin and Anti-Biotin ${ }^{149 S m}$, and ER-Alexa488 and 551 Anti-Alexa488 ${ }^{142 N d}$. Information on the antibodies, metal reporters, and staining 552 concentrations is located in Table S2. 
554

555

556

557

558

559

560

561

562

563

564

565

566

567

568

569

570

571

572

573

574

575

576

577

578

579

580

581

582

583

584

\section{Tissue Staining}

Tissues were sectioned ( $5 \mu \mathrm{m}$ section thickness) from tissue blocks on gold and tantalumsputtered microscope slides. Slides were baked at $70^{\circ} \mathrm{C}$ overnight followed by deparaffinization and rehydration with washes in xylene (3x), 100\% ethanol (2x), 95\% ethanol (2x), 80\% ethanol (1x), 70\% ethanol (1x), and ddH2O with a Leica ST4020 Linear Stainer (Leica Biosystems). Tissues next underwent antigen retrieval by submerging sides in 3-in-1 Target Retrieval Solution ( $\mathrm{pH}$ 9, DAKO Agilent) and incubating at $97^{\circ} \mathrm{C}$ for 40 minutes in a Lab Vision PT Module (Thermo Fisher Scientific). After cooling to room temperature slides were washed in 1x PBS IHC Washer Buffer with Tween 20 (Cell Marque) with $0.1 \%(\mathrm{w} / \mathrm{v})$ bovine serum albumin (Thermo Fisher). Next, all tissues underwent two rounds of blocking, the first to block endogenous biotin and avidin with an Avidin/Biotin Blocking Kit (Biolegend). Tissues were then washed with wash buffer and blocked for 1 hour at room temperature with 1x TBS IHC Wash Buffer with Tween 20 with $3 \%(\mathrm{v} / \mathrm{v})$ normal donkey serum (Sigma-Aldrich), $0.1 \%(\mathrm{v} / \mathrm{v})$ cold fish skin gelatin (Sigma Aldrich), $0.1 \%(\mathrm{v} / \mathrm{v})$ Triton X-100, and $0.05 \%(\mathrm{v} / \mathrm{v})$ Sodium Azide. The first antibody cocktail was prepared in 1x TBS IHC Wash Buffer with Tween 20 with 3\% (v/v) normal donkey serum (Sigma-Aldrich) and filtered through a $0.1 \mu \mathrm{m}$ centrifugal filter (Millipore) prior to incubation with tissue overnight at $4^{\circ} \mathrm{C}$ in a humidity chamber. Following the overnight incubation slides were washed twice for 5 minutes in wash buffer. The second day antibody cocktail was prepared as described and incubated with the tissues for 1 hour at $4^{\circ} \mathrm{C}$ in a humidity chamber. Following staining, slides were washed twice for 5 minutes in wash buffer and fixed in a solution of $2 \%$ glutaraldehyde (Electron Microscopy Sciences) solution in low-barium PBS for 5 minutes. Slides were washed in PBS (1x), 0.1 $\mathrm{M}$ Tris at $\mathrm{pH} 8.5(3 \mathrm{x}), \mathrm{ddH} 2 \mathrm{O}(2 \mathrm{x})$, and then dehydrated by washing in $70 \%$ ethanol (1x), $80 \%$ ethanol $(1 \mathrm{x}), 95 \%$ ethanol $(2 \mathrm{x})$, and $100 \%$ ethanol $(2 \mathrm{x})$. Slides were dried under vacuum prior to imaging.

\section{MIBI-TOF Imaging}

Imaging was performed using a MIBI-TOF instrument with a Hyperion ion source. $\mathrm{Xe}^{+}$ primary ions were used to sequentially sputter pixels for a given FOV. The following imaging parameters were used: Acquisition setting: $80 \mathrm{kHz}$, Field size: $500 \mu \mathrm{m}^{2}, 1024 \mathrm{x}$ 


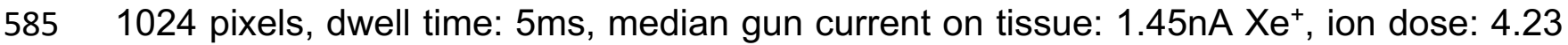
nAmp hours / $\mathrm{mm}^{2}$ for $500 \mu \mathrm{m}^{2}$ FOVs.

587

\section{Low-level Image Processing and Single Cell Segmentation}

589 Multiplexed image sets were extracted, slide background-subtracted, denoised, and 590 aggregate filtered as previously described (Keren et al., 2018; McCaffrey et al., 2020).

591 Nuclear segmentation was performed using an adapted version of the DeepCell CNN 592 architecture (McCaffrey et al., 2020; Valen et al., 2016). To more effectively capture the 593 range of cell shapes and morphologies present in DCIS, we generated two distinct 594 segmentations for each image. The first used a radial expansion of three pixels and a 595 stringent threshold for splitting cells (See Figure S2A, Stroma Parameters). The second 596 used a radial expansion of one pixel and lenient threshold for splitting cells (Epithelial 597 Parameters). We combined these masks together using a post-processing step which 598 gave preference to the epithelial segmentation mask, overriding and stromal-mask599 detected objects in the same area. Smaller cells identified by the stromal settings and 600 missed in the epithelial settings were combined to the final cell mask. A cell nuclei ("Nuc") 601 channel combining $\mathrm{HH} 3$ and endogenous phosphorous $(\mathrm{P})$ signal was made to increase signal robustness for nuclei detection.

603

\section{Single Cell Phenotyping and Composition}

605 Single cell data was extracted for all cell objects and area normalized. Single cell data 606 was linearly scaled by average cell area across the cohort and asinh-transformed with a 607 co-factor of 5. All mass channels were scaled to 99.9th percentile. In order to assign each 608 cell to a lineage, the FlowSOM clustering algorithm was used in iterative rounds with the 609 Bioconductor "FlowSOM" package in R (Van Gassen et al., 2015). The first clustering 610 round separated cells into 100 clusters that were subsequently merged into one of five 611 major cell lineages (tumor, myoepithelial, fibroblast, endothelial, immune) based on the 612 clustering nodes. Proper lineage assignments were ensured by overlaying Flowsom 613 cluster identity with lineage-specific markers. Supervised lineage reassignment was 614 performed where needed. Immune cells were subclustered again to delineate B cells, $615 \mathrm{CD}^{+} \mathrm{T}$ cells, CD8 ${ }^{+} \mathrm{T}$ cells, monocytes, MonoDC cells, DC cells, macrophages, 
616 neutrophils, mast cells, double-negative CD4-CD8- T cells (dnT cells), and HLADR ${ }^{+}$APC

617 cells. CD45+-only immune cells were annotated as 'immune other.' Tumor and fibroblast

618 cells were similarly clustered again to reveal phenotypic subsets, as shown in Figure S2.

619 Altogether, we assigned $94 \%(n=127,451$ of 134,631$)$ of cells to 16 subsets, with the

620 remaining nucleated cells with absent or very low levels of lineage markers assigned as

621 "other". The relative abundance of all major lineages was determined out of total cells per

622 FOV and the relative frequency of cell subsets were determined out of total cells of a

623 given lineage, per FOV.

624

625

\section{Region Masking}

626 Region masks were generated to define histologic regions of each FOV including the 627 epithelium, stroma, myoepithelial (periductal) zone, and duct, which was further 628 subdivided into the duct edge, duct mid, and duct core. We removed gold-positive area 629 which marked regions of bare slide from holes in the tissue, providing an accurate 630 measurement of tissue area. This area measurement could be used to calculate cellular density in specific histologic regions, e.g., fibroblast density in the stroma, which was

632 critical to normalize the observed cell abundances by how much tissue of a specific type 633 was sampled, and prevent bias based on how much tumor vs stroma the FOV covered.

634 The epithelial mask was first generated though merging ECAD and PanCK signal and 635 applying smoothing and radial expansion to incorporate the myoepithelial zone, and the 636 inside of ducts were filled. The stromal mask included all image area outside of the 637 epithelial mask. Duct masks were generated through the erosion of the epithelial masks 638 by 25 pixels. The myoepithelial mask was generated by subtracting the duct mask from 639 the epithelial mask. Duct edge, duct mid, and duct core masks (Figure 3I) were generated 640 by eroding the duct mask by subsequent 100-pixel increments.

641

\section{Protein and Cellular Spatial Enrichment Analyses}

643 A spatial enrichment approached was used as previously described (Keren et al., 2018, 644 2019; McCaffrey et al., 2020) to identify patterns of protein enrichment or exclusion across 645 all protein pairs. HH3 was excluded from the analysis. For each pair of markers, $\mathrm{X}$ and $\mathrm{Y}$, 646 the number of times cells positive (normalized expression $>0.25$ ) for protein $X$ was within 
647 a $\sim 50$ um radius of cells positive for protein $Y$ was counted. A null distribution was 648 produced by performing 100 bootstrap permutations where the locations of cells positive for protein $Y$ were randomized. A z-score was calculated comparing the number of true

650 cooccurrences of cells positive for protein $X$ and $Y$ relative to the null distribution.

651 Importantly, symmetry is assumed: the values of when calculating the spatial enrichment

652 of protein $X$ close to protein $Y$ are the same as with protein $Y$ close to protein $X$. For each

653 pair of proteins $X$ and $Y$ the average $z$-score was calculated across all DCIS FOVs.

654

To analyze cellular associations with the myoepithelium, the distances between all cell centroids to the nearest perimeter location of the myoepithelium mask (described above) were calculated. To quantity cell type spatial interactions, the mean distances

657 between cell centroids for all cell phenotype pairs (self-self pairs excluded) were 658 calculated per region.

659 Cell neighborhoods were produced by first generating a cell neighbor matrix, where each row represents an index cell, and the columns indicate the relative frequency of each cell phenotype within an 36um radius of the index cell. Next the neighbor matrix was clustered to 10 clusters using k-means clustering. Neighborhood cellular profile was determined by assessing the mean prevalence of each cell phenotype in the index cells' 36um radius, while functional marker expression was determined by assessing mean marker expression by the index cells assigned to each neighborhood cluster.

\section{DCIS UMAP Visualization}

668 UMAP embeddings were determined for all DCIS tumors (pure, synchronous, primary 669 and recurrent) using the $R$ implementation (Mclnnes et al., 2020) with the following 670 parameters: $n \_$neighbors $=15$, min dist $=0.1$ and the following markers: PanCK, CK7, 671 CK5, ECAD, VIM, ER, HER2, AR, CD31, SMA, CD45, HLADR, CD68, CD11c, CD14, 672 CD20, CD3, CD4, CD8, MPO, Tryptase.

674 EMT GSEA

675 To identify genes and pathways associated to EMT, MIBI-identified DCIS vimentin high 676 vs low samples were selected, and the epithelial fraction of an adjacent tissue section 677 was analyzed by LCM-RNAseq (Vim high, $n=26$; Vim low, $n=32$ ). DESeq2 R package 
678 (version 1.30.0) was used for data normalization and differential expression analysis.

679 Results were sorted by decreasing log fold change and the ranked list was subjected to

680 GSEA against C2 curated dataset of molecular signature database

681 (MSigDB)(Subramanian et al., 2005). P values were corrected for multiple comparisons

682 by using Benjamini-Hochberg method and terms with $\mathrm{p}$ adj $<0.05$ were considered.

683

684 ECM Gene Analysis

685 To analyze extracellular matrix components by gene expression, an extracellular matrix 686 gene signature (GO extracellular matrix structural constituent, GO:0030021) was 687 downloaded from GSEA website and used to compare MIBI-identified samples with the 688 top and bottom quartiles of cancer associated fibroblast density in the stroma. Stromal 689 LCM-RNAseq samples were used for this analysis. Raw reads were normalized with 690 DESeq2 R package (version 1.30.0)(Anders and Huber, 2010) and a paired T-test was 691 compared to the log2 ratio of group means to generate the volcano plot.

692

\section{Myoepithelial Continuity and Thickness Analysis}

694 To define a window of myoepithelial signal quantitation, we used a topology-preserving 695 operation to define a curve 5 pixels out from the epithelial mask edge (see Region Masking) and a curve 30 pixels in from the epithelium mask edge, and we defined those pixels in between these two curves as the myoepithelium mask. We subdivided the outer curve into 5-pixel long arc-segments, and for each point on the outer edge in between two segments, found the nearest point on the inner edge, dividing the myoepithelium into a string of quadrilaterals or "wedges". Wedges are then subdivided each wedge along the 701 in-out (of the epithelium) axis into 10 segments. Wedges are merged when both their 702 combined inner and outer edges has an arc-length less than 15 pixels.

703

We took pre-processed (background subtracted, de-noised) SMA pixels within the mesh and smoothed them with a Gaussian blur of radius of 1 . We then calculated the density of SMA signal within each mesh-segment as the mean pixel value of smoothed SMA within that mesh-segment. This density was then binarized to create a SMApositivity mesh, using a threshold of 0.5 (density $>0.5$ as positive). 
The percentage of duct perimeter covered by myoepithelium was calculated by assigning an "SMA-present" variable to each wedge, "0" if no mesh-segments in the wedge were positive for SMA, and "1" otherwise. Each wedge is weighted by its area relative to the myoepithelium area. The sum over all wedges of the product of the "SMApresent" variable and the weight was defined as the percent perimeter SMA positivity.

713 The average (non-zero) thickness of the myoepithelium for each duct was calculated by

714 finding the weighted average "wedge thickness" for SMA-positive wedges ("SMApresent" was 1). The wedge thickness was calculated as the distance between the innermost and outer-most positive mesh-segments. The positive wedges were weighted by their area relative to the total area of positive wedges. duct equal to the total area of the duct myoepithelium divided by the sum of the total areas of all myoepithelium in the image that met a minimum size filter of 7500 pixels.

722

\section{Myoepithelial Pixel Clustering Analysis}

724 Pre-processed (background subtracted, de-noised) images were first subset for pixels within the myoepithelium mask. Pixels within the myoepithelium mask were then further subset for pixels with SMA expression greater than 0 . For all $\mathrm{SMA}^{+}$pixels within the

727 myoepithelium mask, a Gaussian blur was applied using a standard deviation of 1.5 for the Gaussian kernel. Pixels were normalized by their total expression, such that the total

729 expression of each pixel was equal to 1. A 99.9\% normalization was applied for each 730 marker. Pixels were clustered into 100 clusters using FlowSOM (Van Gassen et al., 2015) 731 based on the expression of 6 markers: PanCK, CK5, Vimentin, ECAD, CD44, and CK7.

732 The average expression of each of the 100 pixel clusters was found and the z-score for 733 each marker across the 100 pixel clusters was computed. All z-scores were capped at 3, such that the maximum z-score was 3. Using these z-scored expression values, the 100 735 pixel clusters were hierarchically clustered using Euclidean distance into 6 metaclusters. $736 \mathrm{SMA}^{+}$pixels that were negative for the 6 markers used for FlowSOM were annotated as 737 the SMA-only metacluster, resulting in a total of 7 metaclusters. These metaclusters were 
738 mapped back to the original images to generate overlay images colored by pixel 739 metacluster.

\section{Collagen Morphometrics}

742 To identify collagen fibers the background-removed Col1 images are first preprocessed:

743 Col1 pixel intensities were capped at 5 and gamma transformed (1 of 2), and contrast 744 enhanced. Images are then blurred via gaussian with sigma of 2. While this enhances 745 fidelity, it gives less clear '0-borders'. This is mitigated by generating a '0-region' mask and setting all values to 0 in that region. Then, highly localized contrast enhancement is applied. Raw fiber signal intensity can vary greatly within a FOV, so this step helps to enhance locally recognizable, but globally dim fiber candidates. After this process, contrast is globally enhanced via a reverse gamma transformation (2 of 2).

Collagen fiber objects are generated by watershed segmentation on the preprocessed images. An adaptive thresholding method was developed to appreciate variability in total image intensities across the large dataset. A dilated and eroded version of each preprocessed image was produced and subjected to multiotsu thresholding. For thin fibers, the higher watershed region is set to everywhere where the eroded image has greater intensity than the highest multiotsu threshold for the eroded image, while the lower watershed region is set to everywhere where the dilated image has lower intensity than the highest multiotsu threshold for the eroded image. For thick fibers, the same procedure is performed, except the lower watershed region uses the middle multiotsu threshold for

759 the dilated image. Elevation maps for watershed are generated via the sobel gradient of

760 a blurred version of the preprocessed images. Once objects are extracted and 761 segmented, length, global orientation, perimeter, and width are computed for each object.

762 Objects which cover low intensity regions of the image are treated as preprocessing 763 artifacts and are not included in averaging.

For fiber alignment scoring, fibers are filtered for elongated shape (length > $7652 *$ width), and alignment is scored as the normalized total paired square difference over 766 its $k$ nearest neighbors ( $k=4$ was chosen). To accommodate for the elongated shape of 767 these object, K-nearest neighbors were computed with the 'ellipsoidal membrane 
768 distance' (EM distance), which is the Euclidean centroid distance minus the portion of said distance that lies within the ellipse representation of the object.

\section{Cibersort Analysis}

772 CIBERSORTx (CSx)(Newman et al., 2019) was used to infer the immune fraction in LCM-

773 SMART3SEQ samples. We first generated a tissue resident immune cell signature matrix

774 by using a published breast cancer scRNAseq dataset, downloaded from Gene 775 Expression Omnibus database (GEO data repository accession numbers GSE114727, 776 GSE114725)(Barrett et al., 2013). Normalized counts were obtained by using Seurat R 777 package (version 3.2.0). The resultant signature matrix contained 3484 genes and 778 allowed to resolve different immune cell types, including B, CD8 T, CD4 T, NKT, NK, mast 779 cells, neutrophils, monocytes, macrophages and dendritic cells. The signature matrix was 780 first in-silico validated. In order to test the accuracy of the signature matrix, a set of 781 samples from the same scRNAseq dataset was reserved to build a synthetic matrix of 782 bulk RNAseq data. By mixing different proportion of single cells transcripts, the synthetic 783 bulk was used to analyze the correlation between known vs obtained cell proportions by 784 CSx. Pearson's coefficient was above 0.75 in all of the cases, most of them above 0.9. 785 Therefore, we used the aforementioned matrix to deconvolve the LCM-RNAseq samples and to compare CSx-estimated cell abundance with MIBI-identified cell types.

\section{Prediction of recurrence}

789 To predict recurrence, we identified patients in the cohort with follow-up data 790 demonstrating carcinoma recurrence $(n=12)$, invasive recurrence $(n=19)$, or at least 11 791 years without recurrence $(n=47)$. For each patient, a vector of summary statistics was 792 generated from MIBI data using only images derived from the original lesion. The cohort 793 was split into training and test sets (80/20\%); all model optimization and predictor 794 selection used only the training set. Any missing values were replaced with the set's 795 predictor mean. Predictors with $<12$ unique values in the training set were dropped from 796 the analysis. Two-class random forest probability models (ranger package)(Wright and 797 Ziegler, 2017) were trained to discriminate recurrence versus non-recurrence, and 798 invasive recurrence versus non-recurrence. Hyperparameters were tuned to minimize 
799 out-of-bag error. One tuned hyperparameter was predictor subset selection by

800 correlation thresholding: predictors were ranked in importance by performing a KS test

801 between recurrence and non-recurrence. Greater importance was placed on predictors

802 with lower p-values, with ties broken by weighting predictors with greater coefficients of

803 variance (CV). All predictors were correlated (Spearman method) and correlations were

804 thresholded (invasive $r>|0.5|$, all recurrence $r>|0.6|$ ). For each group of correlated

805 predictors above a given threshold, only the highest-ranked predictor was used in the

806

807

808

809

810

811

812

813

814

815

816

\section{7}

818

819

820

821

822

823

824

825

826

827

828

829 model. The optimized random forest model was evaluated on the test set and a receiver operating characteristic (ROC) curve was generated (pROC package)(Robin et al., 2011) using the model's assigned probability scores. Area under the curve (AUC) was calculated with $95 \%$ confidence intervals, determined by bootstrapping. Each predictor's importance was evaluated in the model by its Gini index. Similarly, two-class random forest probability models were also trained using only clinical parameters as predictors (age, mammograph density, tumor grade, and tumor necrosis) without subset selection. For the MIBI-based predictions, an optimal probability threshold was selected by the Youden method to assign predicted class to the test set, and Kaplan-Meier curves were calculated (survival package)(Therneau and Grambsch, 2000).

\section{Statistical Analysis}

All statistical analyses were performed using GraphPad Prism software or in R. Grouped data is presented with individual sample points throughout, and where not applicable, data is presented as a mean with standard deviation. For determining significance, grouped data was first tested for normality with the D'Agostino \& Pearson omnibus normality test. Normally distributed data was compared between two groups with the twotailed Student's T-test. Non-normal data was compared between two groups using the Mann-Whitney Test. Multiple groups were compared using the Dunn's Multiple Comparison Test.

\section{Software}

Image processing was conducted with Matlab 2016a and Matlab 2019b. Statistical analysis was conducted in Graphpad Prism. Data visualization and plots were generated 
830 in R with ggplot and pheatmap packages, in Graphpad Prism, and in Python using the scikitimage, matplotlib, and seaborn packages. Representative images were processed

832 in Adobe Photoshop. Schematic visualizations were produced with Biorender. R

833 packages for GSEA: AnnotationDbi, 1.52 .0 \& org.Hs.eg.db, 3.12.0, clusterProfiler, version 3.19.0, for GSEA msigdbr, version '7.2.1', for C2 curated datasets. Python packages for spatial enrichment analysis and collagen morphometrics: sckikit-image, pandas, numpy, xarray, scipy, statsmodels.

\section{Data and Code Availability}

839 All custom code used to analyze data will be made available through a Github repository and all processed images and annotated single cell data will be made available on a Human Tumor Atlas Network public repository.

\section{Author Contributions}

845 TR conceived the study design, performed experiments, analyzed data, and wrote the 846 manuscript with MA. DG developed the classifier model and performed related analyses. 847 CCL developed the myoepithelial pixel clustering approach and performed related analyses. SHS. processed the LCM-RNAseq data and BRG performed all RNAseq analyses. EFM assisted with data analysis with AK, LK, and SV. N.F.G. assisted with

850 image segmentation. $A B$ developed and performed the myoepithelial morphology

851 analyses and AK performed the collagen morphology analyses. GAC, DJV, KD assisted 852 with cohort design and patient sample preparation, and SS performed pathological 853 review, and SV and ZK assisted with immunohistochemistry. SEH, SCB, RBW and MA 854 supervised the work.

855

\section{Acknowledgements}

857 The authors thank the HTAN Consortium for the intellectual and collaborative support of 858 this work. We would like to thank Pauline Chu and the Stanford Human Histology Core 859 for providing technical assistance. TR was supported by the American Cancer Society 
861 Grant 5 T32 AI07290-33. DRG was supported by the Bio-X Stanford Interdisciplinary 862 Graduate Fellowship. CCL was supported by the Stanford Graduate Fellowship. RBW 863 was supported by R01CA193694 and U2C CA233254. MA was supported by 1-DP5864 OD019822. SCB and MA were jointly supported by 1R01AG056287 and 1R01AG057915, 865 1U24CA224309, the Bill and Melinda Gates Foundation, and a Translational Research 866 Award from the Stanford Cancer Institute.

\section{Conflicts of Interest}

M.A. and S.C.B. are inventors on patent US20150287578A1. M.A. and S.C.B. are board members and shareholders in lonPath Inc. T.R. and E.F.M. have previously consulted for

871 IonPath Inc.

\section{References}

Afghahi, A., Forgó, E., Mitani, A.A., Desai, M., Varma, S., Seto, T., Rigdon, J., Jensen, K.C., Troxell, M.L., Gomez, S.L., et al. (2015). Chromosomal copy number alterations for associations of ductal carcinoma in situ with invasive breast cancer. Breast Cancer Res. 17, 108. Invasion in Breast Tumors Identified by Topographic Single Cell Sequencing (Cell).

Alaeikhanehshir, S., Engelhardt, E.G., van Duijnhoven, F.H., van Seijen, M., Bhairosing, P.A., Pinto, D., Collyar, D., Sawyer, E., Hwang, S.E., Thompson, A.M., et al. (2020). The impact of patient characteristics and lifestyle factors on the risk of an ipsilateral event after a primary DCIS: A systematic review. Breast Edinb. Scotl. 50, 95-103.

Alcazar, C.R.G.D., Huh, S.J., Ekram, M.B., Trinh, A., Liu, L.L., Beca, F., Zi, X., Kwak, M., Bergholtz, H., Su, Y., et al. (2017). Immune Escape in Breast Cancer During In Situ to Invasive Carcinoma Transition. Cancer Discov. 7, 1098-1115.

Anders, S., and Huber, W. (2010). Differential expression analysis for sequence count data. Genome Biol. 11, R106.

Barsky, S.H., and Karlin, N.J. (2005). Myoepithelial Cells: Autocrine and Paracrine Suppressors of Breast Cancer Progression. J. Mammary Gland Biol. Neoplasia 10, 249-260. Arch. 446, 532-536. 
Betsill, W.L., Rosen, P.P., Lieberman, P.H., and Robbins, G.F. (1978). Intraductal carcinoma. Long-term follow-up after treatment by biopsy alone. JAMA 239, 1863-1867.

896

Buerger, H., Otterbach, F., Simon, R., Poremba, C., Diallo, R., Decker, T., Riethdorf, L., Brinkschmidt, C., Dockhorn-Dworniczak, B., and Boecker, W. (1999). Comparative genomic hybridization of ductal carcinoma in situ of the breast-evidence of multiple genetic pathways. J. Pathol. 187, 396-402.

Conklin, M.W., Eickhoff, J.C., Riching, K.M., Pehlke, C.A., Eliceiri, K.W., Provenzano, P.P., Friedl, A., and Keely, P.J. (2011). Aligned Collagen Is a Prognostic Signature for Survival in Human Breast Carcinoma. Am. J. Pathol. 178, 1221-1232.

Curtis, C., Shah, S.P., Chin, S.-F., Turashvili, G., Rueda, O.M., Dunning, M.J., Speed, D., Lynch, A.G., Samarajiwa, S., Yuan, Y., et al. (2012). The genomic and transcriptomic architecture of 2,000 breast tumours reveals novel subgroups. Nature $486,346-352$.

Erbas, B., Provenzano, E., Armes, J., and Gertig, D. (2006). The natural history of ductal carcinoma <Emphasis Type="Boldltalic">in situ</Emphasis > of the breast: a review. Breast Cancer Res. Treat. 97, 135-144.

Esbona, K., Yi, Y., Saha, S., Yu, M., Doorn, R.R.V., Conklin, M.W., Graham, D.S., Wisinski, K.B., Ponik, S.M., Eliceiri, K.W., et al. (2018). The Presence of Cyclooxygenase 2, Tumor-Associated Macrophages, and Collagen Alignment as Prognostic Markers for Invasive Breast Carcinoma Patients. Am. J. Pathol. 188, 559-573.

Eusebi, V., Feudale, E., Foschini, M.P., Micheli, A., Conti, A., Riva, C., Di Palma, S., and Rilke, F. (1994). Long-term follow-up of in situ carcinoma of the breast. Semin. Diagn. Pathol. 11, 223235.

Foley, J.W., Zhu, C., Jolivet, P., Zhu, S.X., Lu, P., Meaney, M.J., and West, R.B. (2019). Gene expression profiling of single cells from archival tissue with laser-capture microdissection and Smart-3SEQ. Genome Res. 29, 1816-1825.

Fujii, H., Szumel, R., Marsh, C., Zhou, W., and Gabrielson, E. (1996). Genetic progression, histological grade, and allelic loss in ductal carcinoma in situ of the breast. Cancer Res. 56, 5260-5265.

Goswami, S., Sahai, E., Wyckoff, J.B., Cammer, M., Cox, D., Pixley, F.J., Stanley, E.R., Segall, J.E., and Condeelis, J.S. (2005). Macrophages promote the invasion of breast carcinoma cells via a colony-stimulating factor-1/epidermal growth factor paracrine loop. Cancer Res. 65, 52785283.

Hollern, D.P., Swiatnicki, M.R., and Andrechek, E.R. (2018). Histological subtypes of mouse mammary tumors reveal conserved relationships to human cancers. PLoS Genet. 14, e1007135. 
928 Ibrahim, A.M., Moss, M.A., Gray, Z., Rojo, M.D., Burke, C.M., Schwertfeger, K.L., dos Santos, 929 C.O., and Machado, H.L. (2020). Diverse Macrophage Populations Contribute to the 930 Inflammatory Microenvironment in Premalignant Lesions During Localized Invasion. Front. 931 Oncol. 10. Jones, J.L., Shaw, J.A., Pringle, J.H., and Walker, R.A. (2003). Primary breast myoepithelial cells exert an invasion-suppressor effect on breast cancer cells via paracrine down-regulation of MMP expression in fibroblasts and tumour cells. J. Pathol. 201, 562-572.

Keren, L., Bosse, M., Marquez, D., Angoshtari, R., Jain, S., Varma, S., Yang, S.-R., Kurian, A., Van Valen, D., West, R., et al. (2018). A Structured Tumor-Immune Microenvironment in Triple Negative Breast Cancer Revealed by Multiplexed Ion Beam Imaging. Cell 174, 1373-1387.e19.

Kim, M., Chung, Y.R., Kim, H.J., Woo, J.W., Ahn, S., and Park, S.Y. (2020). Immune microenvironment in ductal carcinoma in situ: a comparison with invasive carcinoma of the breast. Breast Cancer Res. BCR 22.

941 Kim, S.Y., Jung, S.-H., Kim, M.S., Baek, I.-P., Lee, S.H., Kim, T.-M., Chung, Y.-J., and Lee, S.H. 942 (2015). Genomic differences between pure ductal carcinoma in situ and synchronous ductal 943 carcinoma in situ with invasive breast cancer. Oncotarget 6, 7597-7607.

944 Kolijn, K., Verhoef, E.I., Smid, M., Böttcher, R., Jenster, G.W., Debets, R., and van Leenders, 945 G.J.L.H. (2018). Epithelial-Mesenchymal Transition in Human Prostate Cancer Demonstrates 946 Enhanced Immune Evasion Marked by IDO1 Expression. Cancer Res. 78, 4671-4679.

947 Lien, H.C., Hsiao, Y.H., Lin, Y.S., Yao, Y.T., Juan, H.F., Kuo, W.H., Hung, M.-C., Chang, K.J., and 948 Hsieh, F.J. (2007). Molecular signatures of metaplastic carcinoma of the breast by large-scale 949 transcriptional profiling: identification of genes potentially related to epithelial-mesenchymal 950 transition. Oncogene 26, 7859-7871.

951 Lin, C.-Y., Tsai, P.-H., Kandaswami, C.C., Lee, P.-P., Huang, C.-J., Hwang, J.-J., and Lee, M.-T. 952 (2011). Matrix metalloproteinase-9 cooperates with transcription factor Snail to induce 953 epithelial-mesenchymal transition. Cancer Sci. 102, 815-827.

954 Linde, N., Casanova-Acebes, M., Sosa, M.S., Mortha, A., Rahman, A., Farias, E., Harper, K., 955 Tardio, E., Reyes Torres, I., Jones, J., et al. (2018). Macrophages orchestrate breast cancer early 956 dissemination and metastasis. Nat. Commun. 9, 21.

957 Liu, Y., West, R., Weber, J.D., and Colditz, G.A. (2019). Race and risk of subsequent aggressive 958 breast cancer following ductal carcinoma in situ. Cancer 125, 3225-3233.

959 Malanchi, I., Santamaria-Martínez, A., Susanto, E., Peng, H., Lehr, H.-A., Delaloye, J.-F., and 960 Huelsken, J. (2012). Interactions between cancer stem cells and their niche govern metastatic 961 colonization. Nature 481, 85-89. 
962 McCaffrey, E.F., Donato, M., Keren, L., Chen, Z., Fitzpatrick, M., Jojic, V., Delmastro, A., 963 Greenwald, N.F., Baranski, A., Graf, W., et al. (2020). Multiplexed imaging of human 964 tuberculosis granulomas uncovers immunoregulatory features conserved across tissue and 965 blood. BioRxiv 2020.06.08.140426.

966 Moen, E., Bannon, D., Kudo, T., Graf, W., Covert, M., and Van Valen, D. (2019). Deep learning 967 for cellular image analysis. Nat. Methods 16, 1233-1246.

968 Morsing, M., Kim, J., Villadsen, R., Goldhammer, N., Jafari, A., Kassem, M., Petersen, O.W., and 969 Rønnov-Jessen, L. (2020). Fibroblasts direct differentiation of human breast epithelial 970 progenitors. Breast Cancer Res. 22, 102.

971 Newburger, D.E., Kashef-Haghighi, D., Weng, Z., Salari, R., Sweeney, R.T., Brunner, A.L., Zhu, 972 S.X., Guo, X., Varma, S., Troxell, M.L., et al. (2013). Genome evolution during progression to 973 breast cancer. Genome Res. 23, 1097-1108.

974 Newman, A.M., Steen, C.B., Liu, C.L., Gentles, A.J., Chaudhuri, A.A., Scherer, F., Khodadoust, 975 M.S., Esfahani, M.S., Luca, B.A., Steiner, D., et al. (2019). Determining cell type abundance and 976 expression from bulk tissues with digital cytometry. Nat. Biotechnol. 37, 773-782.

977 Page, D.L., Dupont, W.D., Rogers, L.W., and Landenberger, M. (1982). Intraductal carcinoma of 978 the breast: follow-up after biopsy only. Cancer 49, 751-758.

979 Pelon, F., Bourachot, B., Kieffer, Y., Magagna, I., Mermet-Meillon, F., Bonnet, I., Costa, A., Givel, 980 A.-M., Attieh, Y., Barbazan, J., et al. (2020). Cancer-associated fibroblast heterogeneity in 981 axillary lymph nodes drives metastases in breast cancer through complementary mechanisms. 982 Nat. Commun. 11, 404.

Peng, J., Wang, X., Ran, L., Song, J., Luo, R., and Wang, Y. (2018). Hypoxia-Inducible Factor $1 \alpha$ Regulates the Transforming Growth Factor $\beta 1 /$ SMAD Family Member 3 Pathway to Promote 985 Breast Cancer Progression. J. Breast Cancer 21, 259-266.

Poola, I., DeWitty, R.L., Marshalleck, J.J., Bhatnagar, R., Abraham, J., and Leffall, L.D. (2005). Identification of MMP-1 as a putative breast cancer predictive marker by global gene

988 expression analysis. Nat. Med. 11, 481-483.

Robin, X., Turck, N., Hainard, A., Tiberti, N., Lisacek, F., Sanchez, J.-C., and Müller, M. (2011). pROC: an open-source package for $\mathrm{R}$ and $\mathrm{S}+$ to analyze and compare ROC curves. BMC Bioinformatics 12, 77.

Ruffell, B., Affara, N.I., and Coussens, L.M. (2012). Differential Macrophage Programming in the

994 Ryser, M.D., Weaver, D.L., Zhao, F., Worni, M., Grimm, L.J., Gulati, R., Etzioni, R., Hyslop, T., Lee, 995 S.J., and Hwang, E.S. (2019). Cancer Outcomes in DCIS Patients Without Locoregional

996 Treatment. JNCI J. Natl. Cancer Inst. 111, 952-960. 
Shani, O., Vorobyov, T., Monteran, L., Lavie, D., Cohen, N., Raz, Y., Tsarfaty, G., Avivi, C.,

998 Barshack, I., and Erez, N. (2020). Fibroblast-derived IL-33 facilitates breast cancer metastasis by

999 modifying the immune microenvironment and driving type-2 immunity. Cancer Res.

1000 Subramanian, A., Tamayo, P., Mootha, V.K., Mukherjee, S., Ebert, B.L., Gillette, M.A., Paulovich, 1001 A., Pomeroy, S.L., Golub, T.R., Lander, E.S., et al. (2005). Gene set enrichment analysis: a

1002 knowledge-based approach for interpreting genome-wide expression profiles. Proc. Natl. Acad.

1003 Sci. U. S. A. 102, 15545-15550.

1004 Therneau, T.M., and Grambsch, P.M. (2000). Modeling Survival Data: Extending the Cox Model 1005 (New York: Springer-Verlag).

1006 Valen, D.A.V., Kudo, T., Lane, K.M., Macklin, D.N., Quach, N.T., DeFelice, M.M., Maayan, I., 1007 Tanouchi, Y., Ashley, E.A., and Covert, M.W. (2016). Deep Learning Automates the Quantitative 1008 Analysis of Individual Cells in Live-Cell Imaging Experiments. PLOS Comput. Biol. 12, e1005177.

1009 Van Gassen, S., Callebaut, B., Van Helden, M.J., Lambrecht, B.N., Demeester, P., Dhaene, T., and 1010 Saeys, Y. (2015). FlowSOM: Using self-organizing maps for visualization and interpretation of 1011 cytometry data. Cytom. Part J. Int. Soc. Anal. Cytol. 87, 636-645.

1012 Wright, M.N., and Ziegler, A. (2017). ranger: A Fast Implementation of Random Forests for High 1013 Dimensional Data in C++ and R. J. Stat. Softw. 77, 1-17.

1014 Yang, M., Li, Z., Ren, M., Li, S., Zhang, L., Zhang, X., and Liu, F. (2018). Stromal Infiltration of 1015 Tumor-Associated Macrophages Conferring Poor Prognosis of Patients with Basal-Like Breast 1016 Carcinoma. J. Cancer 9, 2308-2316.

1017 Zhang, W., Shi, X., Peng, Y., Wu, M., Zhang, P., Xie, R., Wu, Y., Yan, Q., Liu, S., and Wang, J. 1018 (2015). HIF-1 $\alpha$ Promotes Epithelial-Mesenchymal Transition and Metastasis through Direct 1019 Regulation of ZEB1 in Colorectal Cancer. PloS One 10, e0129603.

1020 Zhang, W., Zhang, J., Zhang, Z., Guo, Y., Wu, Y., Wang, R., Wang, L., Mao, S., and Yao, X. (2019). 1021 Overexpression of Indoleamine 2,3-Dioxygenase 1 Promotes Epithelial-Mesenchymal Transition 1022 by Activation of the IL-6/STAT3/PD-L1 Pathway in Bladder Cancer. Transl. Oncol. 12, 485-492.

1023 Zhou, J., Wang, X.-H., Zhao, Y.-X., Chen, C., Xu, X.-Y., sun, Q., Wu, H.-Y., Chen, M., Sang, J.-F., Su, 1024 L., et al. (2018). Cancer-Associated Fibroblasts Correlate with Tumor-Associated Macrophages 1025 Infiltration and Lymphatic Metastasis in Triple Negative Breast Cancer Patients. J. Cancer 9, 1026 4635-4641. 